\title{
Point Defects and Localized Excitons in 2D $\mathrm{WSe}_{2}$
}

Yu Jie Zheng ${ }^{1,2, \dagger}$, Yifeng Chen ${ }^{2, \dagger}$, Yu Li Huang ${ }^{1,3}$, Pranjal Kumar Gogoi ${ }^{1}$, Ming-Yang Li $^{4}$, Lain-Jong Li $i^{4}$, Paolo E. Trevisanutto ${ }^{2}$, Qixing Wangl, Stephen J. Pennycook ${ }^{5, *}$, Andrew T. S. Wee ${ }^{1,2, *}$, Su Ying Quek $k^{1,2, *}$

${ }^{1}$ Department of Physics, National University of Singapore, 2 Science Drive 3, 117542 , Singapore

${ }^{2}$ Centre for Advanced 2D Materials, National University of Singapore, Block S14, Level 6, 6 Science Drive 2, 117546, Singapore

${ }^{3}$ Institute of Materials Research \& Engineering (IMRE), A*STAR (Agency for Science, Technology and Research), 2 Fusionopolis Way, Innovis, 138634, Singapore

${ }^{4}$ Physical Sciences and Engineering, King Abdullah University of Science and Technology, Thuwal, 23955-6900, Saudi Arabia

${ }^{5}$ Department of Materials Science \& Engineering, National University of Singapore, 9 Engineering Drive 1, Singapore 117575 


\section{ABSTRACT}

Identifying the point defects in 2D materials is important for many applications. Recent studies have proposed that $\mathrm{W}$ vacancies are the predominant point defect in $2 \mathrm{D} \mathrm{WSe}$, in contrast to theoretical studies, which predict that chalcogen vacancies are the most likely intrinsic point defects in transition metal dichalcogenide semiconductors. We show using first principles calculations, scanning tunneling microscopy (STM) and scanning transmission electron microscopy experiments, that $\mathrm{W}$ vacancies are not present in our CVD-grown $2 \mathrm{D} \mathrm{WSe}_{2}$. We predict that O-passivated Se vacancies $\left(\mathrm{O}_{\mathrm{Se}}\right)$ and $\mathrm{O}$ interstitials $\left(\mathrm{O}_{\text {ins }}\right)$ are present in $2 \mathrm{D} \mathrm{WSe}$, because of facile $\mathrm{O}_{2}$ dissociation at $\mathrm{Se}$ vacancies, or due to the presence of $\mathrm{WO}_{3}$ precursors in CVD growth. These defects give STM images in good agreement with experiment. The optical properties of point defects in $2 \mathrm{D} \mathrm{WSe}_{2}$ are important because single photon emission (SPE) from $2 \mathrm{D} \mathrm{WSe}$ has been observed experimentally. While strain gradients funnel the exciton in real space, point defects are necessary for the localization of the exciton at length scales that enable photons to be emitted one at a time. Using state-of-the-art GW-Bethe-Salpeter-equation calculations, we predict that only $\mathrm{O}_{\text {ins }}$ defects give localized excitons within the energy range of SPE in previous experiments, making them a likely source of previously observed SPE. No other point defects (Ose, Se vacancies, W vacancies and Sew antisites) give localized excitons in the same energy range. Our predictions suggest ways to realize SPE in related 2D materials and point experimentalists toward other energy ranges for SPE in $2 \mathrm{D} \mathrm{WSe} 2$.

KEYWORDS: defects, 2D materials, $\mathrm{WSe}_{2}, \mathrm{MoS}_{2}$, optical properties, single photon emission, GW-BSE 
Elucidating the nature and properties of point defects in materials is of fundamental importance. For 2D materials, in particular, complete elimination of defects in growth processes is still a challenging task, and the high surface-to-volume ratio also renders 2D materials more susceptible to unintentional reactions with chemicals in the environment. The implications of point defects on the properties of $2 \mathrm{D}$ materials are multifold. It has been found that removing sulphur vacancies $\left(\mathrm{S}_{\mathrm{vac}}\right)$ in $\mathrm{MoS}_{2}$ by reaction with thiol molecules can result in much improved optical quality. ${ }^{1}$ This observation can be attributed to the gap states in $S_{\text {vac }}$ and the fact that $S_{\text {vac }}$ are susceptible to adsorption of contaminants from the environment. On the other hand, defects in transition metal dichalcogenides (TMDs) can have a large impact on spin transport properties in graphene/TMD heterostructures. ${ }^{2}$ Point defects, together with strain gradients, are also believed to play an important role in single photon emission (SPE) from $2 \mathrm{D} \mathrm{WSe}_{2}, 3,4$ which has been observed by several experimental groups. ${ }^{5-10}$

It is interesting to ask why most successful SPE experiments in TMD have utilized $\mathrm{WSe}_{2}$, rather than the more common TMD, $\mathrm{MoS}_{2}$. It has been suggested that the reason for this phenomenon is that $\mathrm{WSe}_{2}$ hosts low-lying dark exciton states, which can increase the lifetime of the excitons and assist in the funneling of excitons to point defects. However, several theoretical predictions suggest that 2D MoS 2 may also host dark exciton states that are lower in energy ${ }^{11}$ or degenerate ${ }^{12}$ with the bright exciton. On the other hand, $2 \mathrm{D} \mathrm{WSe}_{2}$ has been found to have excellent optical quality compared to $\mathrm{MoS}_{2 .}{ }^{13}$ These differences suggest that the defect structure in $\mathrm{WSe}_{2}$ is fundamentally different from that in $\mathrm{MoS}_{2}$. We remark that most of the optical experiments have been performed on exfoliated samples rather than samples grown by chemical vapour deposition (CVD), where grain boundaries can also be present. Scanning tunnelling microscopy (STM) and first principles density functional theory (DFT) calculations 
have shown that sulphur vacancies $S_{\text {vac }}$ are the predominant point defects in mechanically exfoliated $\mathrm{MoS}_{2}$ monolayers. ${ }^{14}$ First principles calculations have also predicted that the chalcogen vacancy $\left(\mathrm{Se}_{\mathrm{vac}}\right)$ has the lowest formation energy among the intrinsic point defects in single layer (SL)-WSe $2,{ }^{15}$ and it is therefore natural to ask if $\mathrm{Se}_{\mathrm{vac}}$ could be the point defect giving rise to localized excitons in SL-WSe2. However, recent electron-beam irradiation experiments on $\mathrm{SL}-\mathrm{WSe} 2$ have shown that the $\mathrm{Se}_{\mathrm{vac}}$ created by the electron beam gives a broad emission peak extending to at least $0.2 \mathrm{eV}$ below the A peak, ${ }^{16}$ while many-electron GW-BSE calculations on $\mathrm{Se}_{\mathrm{vac}}$ in $\mathrm{SL}-\mathrm{WSe}{ }_{2}{ }^{17}$ and $\mathrm{S}_{\mathrm{vac}}$ in $\mathrm{MoS}_{2}{ }^{18}$ predict broad optical absorption spectra arising from chalcogen vacancies. In contrast, the SPE previously observed from SL-WSe2 correspond to localized excitons $\sim 45-100 \mathrm{meV}$ below the free exciton A peak. ${ }^{5-10}$ This implies that $\mathrm{Se}_{\mathrm{vac}}$ are not responsible for the localized excitons in $\mathrm{SL}-\mathrm{WSe} 2 .{ }^{5-10}$ Instead, it has been suggested, based on STM images, that $\mathrm{W}$ vacancies $\left(\mathrm{W}_{\mathrm{vac}}\right)$ are the predominant point defects in SL-WSe2, and are responsible for localized SPE. ${ }^{19}$

In this work, we use a combination of first principles calculations, scanning tunneling microscopy (STM) and scanning transmission electron microscopy (STEM) experiments to investigate the point defects in $\mathrm{SL}_{-} \mathrm{WSe}$. Our calculations show that W vacancies are not energetically favored, and the experiments show that $\mathrm{W}$ vacancies are not present in our CVD-grown samples. Instead, we predict that $\mathrm{Se}_{\mathrm{vac}}$ have the lowest formation energy among all intrinsic defects in SL-WSe2. However, O-substituted Se vacancy $\left(\mathrm{Ose}_{\mathrm{se}}\right)$ and $\mathrm{O}$ interstitial defects $\left(\mathrm{O}_{\text {ins }}\right)$ are favored due to facile $\mathrm{O}_{2}$ dissociation at $\mathrm{Se}_{\text {vac }}$ sites at room temperature, or the use of metal oxide precursors for CVD-grown samples. Simulated STM images of these O defects agree well with our experimental STM images and the aforementioned STM results. ${ }^{19}$ Unlike the case for $\mathrm{MoS}_{2},{ }^{14}$ STM images do not reveal the presence of any chalcogen vacancies. It is interesting to note 
that our findings are consistent with the recent observation of O-substituted chalcogen vacancies in $2 \mathrm{D} \mathrm{MoSe} 2$ and $2 \mathrm{D} \mathrm{WS}_{2} .{ }^{20}$ We note that the $2 \mathrm{D} \mathrm{MoSe} 2$ in Ref. 20 was grown from elemental sources. According to our theoretical calculations, the propensity for $\mathrm{O}_{2}$ to dissociate at chalcogen vacancies is related to the work function of the $2 \mathrm{D}$ materials, and the energy barrier for $\mathrm{O}_{2}$ dissociation at chalcogen vacancies should be largest for $\mathrm{MoS}_{2}$, smallest for $\mathrm{WSe}_{2}$, and intermediate for $\mathrm{MoSe}_{2}$ and $\mathrm{WS}_{2}$. Our results therefore contribute to a unified picture of the properties of chalcogen defects in TMD semiconductors. $^{21}$

The passivation of $\mathrm{Se}_{\mathrm{vac}}$ gap states explains the superior optical quality of $2 \mathrm{D} \mathrm{WSe}$, compared to 2D MoS 2 , and facilitates the observation of SPE from other defects. We use state-of-the-art many-body perturbation theory (GW-BSE calculations) to study the nature and energies of excitons at the experimentally observed point defects. This theoretical study is motivated by the recent SPE experiments in $2 \mathrm{D} \mathrm{WSe} 2$, and also by the fact that identifying the source of SPE is important, but experimentally challenging. We find that only $\mathrm{O}_{\text {ins }}$ gives rise to localized excitons within the energy range for SPE previously observed in SL-WSe $2 .{ }^{5-10}$ Our experimental investigations also reveal the presence of Sew antisite defects, which is consistent with our theoretical predictions for typical Se-rich growth conditions. Like $\mathrm{W}_{\mathrm{vac}},{ }^{17} \mathrm{Sew}$ gives rise to many sub-bandgap exciton peaks that are much lower in energy than the A peak.

Taken together, we have used a combination of theory and two different experimental techniques to elucidate the nature of point defects in $\mathrm{WSe}_{2}{ }^{21} \mathrm{We}$ show that the chalcogen vacancies in $\mathrm{SL}_{-} \mathrm{WSe}_{2}$ are passivated by atomic $\mathrm{O}$, in contrast to sulphur vacancies in $\mathrm{SL}-\mathrm{MoS}_{2}$, explaining the superior optical quality of $\mathrm{WSe}_{2}$, compared to mechanically exfoliated $\mathrm{MoS}_{2}$ samples. We also predict the optical properties of the experimentally observed point defects, which not only shed light on 5 
recent SPE experiments, but also suggest ways to create quantum emitters in related $2 \mathrm{D}$ materials as well as point experimentalists toward other energy ranges for SPE in 2D $\mathrm{WSe}_{2}$.

\section{RESULTS AND DISCUSSION}

Fig. 1a shows the formation energy of intrinsic defects in SL-WSe 2 on graphite, computed using density functional theory (DFT; see Methods and SI). Subscripts 'ad', 'vac' and 'int' refer to adatoms, vacancies and interstitials, respectively. $2 \mathrm{Se}_{\mathrm{vac}}$ refers to a double Se vacancy at the same site and Sew refers to an antisite defect with Se substituting W. As Se and $\mathrm{W}$ adatom and interstitial defects can be removed by annealing, they are not our focus here. Of all the intrinsic point defects we have studied for SL-WSe2, we find that for both W-rich and Se-rich conditions, $\mathrm{Se}_{\mathrm{vac}}$ has the lowest formation energy (2.2-2.8 eV), while the defects with the next smallest formation energies are $\mathrm{Se}, \mathrm{W}_{\mathrm{vac}}$ and $2 \mathrm{Se}_{\mathrm{vac}}$, with formation energies greater than $3.6 \mathrm{eV}$. Based on these defect formation energies, we can conclude that $\mathrm{Se}_{\mathrm{vac}}$ would by far be the most abundant intrinsic point defect in $\mathrm{SL}-\mathrm{WSe}_{2}$. In Fig. S1, we provide information on intrinsic point defects with formation energy greater than $6 \mathrm{eV}$. The atomic structures of all intrinsic defects are shown in Fig. S2. It is interesting to note that our predictions on the formation energy for intrinsic point defects in graphite-supported SL-WSe 2 are in close agreement with those for isolated $\mathrm{SL}-\mathrm{WSe}_{2}$ (Table $\mathrm{S} 1$ ). This is attributed to weak interactions between $\mathrm{WSe}_{2}$ and graphite, and the fact that the strain on graphitesupported $\mathrm{WSe}_{2}$ is very small $(0.6 \%)$. Except for the Se adatom, gap states are evident in the densities of states (DOS) of all intrinsic defects (Fig. S3). The DOS computed using the hybrid HSE06 exchange-correlation functional is shown for $\mathrm{Se}_{\mathrm{vac}}$, Sew and $\mathrm{W}_{\text {vac }}$ in Fig. 1b-d. 

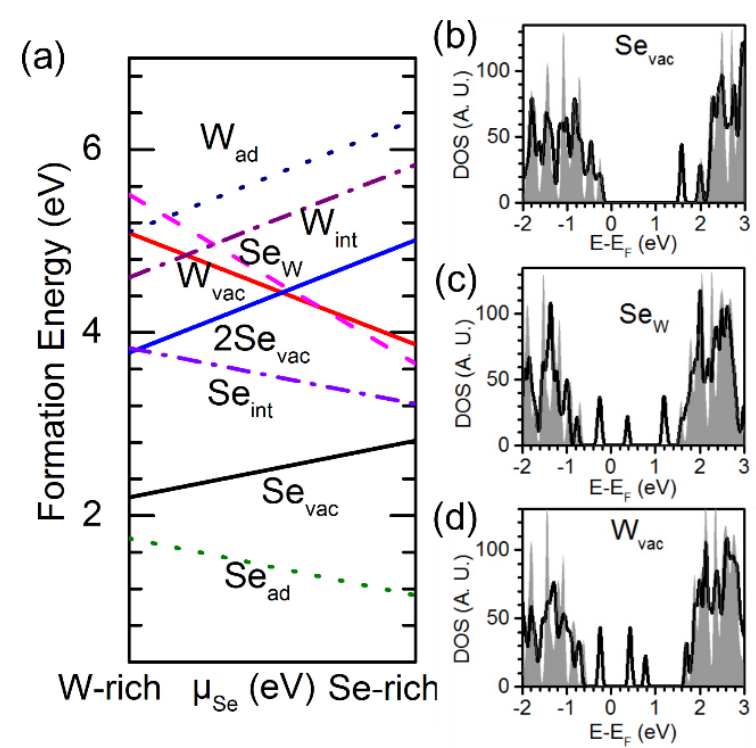

Figure 1. Intrinsic defects in SL-WSe2. (a) Formation energies of the intrinsic defects for $\mathrm{SL}_{-} \mathrm{WSe}_{2}$ on graphite. Solid lines denote vacancies, dashed lines substitutional (antisite) defects, dotted lines adatoms, and dotted-dashed lines intercalated atoms. (bd) DOS of the $5 \times 5 \mathrm{WSe}_{2}$ supercell with intrinsic defects. The DOS plots are computed using the HSE06 hybrid exchange-correlation functional. Gray shading: DOS of perfect WSe2, aligned using the $1 \mathrm{~s}$ core level of the $\mathrm{W}$ atoms furthest from the defect.

In the above discussion, we have established from our DFT calculations that just like in other TMD semiconductors, ${ }^{15}$ chalcogen vacancies are the predominant intrinsic point defect in SL-WSe2, and that all the intrinsic point defects considered here have gap states. However, as we shall show below, scanning tunnelling spectroscopy (STS) shows that all the point defects in our scanning tunnelling microscopy (STM) images have no gap states, an observation that is consistent with previous reports. ${ }^{19}$ On the other hand, 2D materials are susceptible to unintentional chemical interactions with the environment. In ambient conditions, one of the most likely sources of contamination is molecular oxygen, $\mathrm{O}_{2}$. Our DFT calculations show that the most stable of the O-related defects are dissociated $\mathrm{O}_{2}$ bound at the $2 \mathrm{Se}_{\mathrm{vac}}$ site, $\mathrm{O}$ bound to $\mathrm{Se}_{\mathrm{vac}}\left(\mathrm{O}_{\mathrm{se}}\right.$; Fig. $\left.2 \mathrm{~d}\right), \mathrm{O}$ in 
an interstitial site within the $\mathrm{WSe}_{2}$ lattice ( $\mathrm{O}_{\text {ins }}$; Fig. 2f), and $\mathrm{O}$ adsorbed on $\mathrm{Se}\left(\mathrm{O}_{\text {ad }}\right.$; Fig. 2h). All of these defects have no gap states, in contrast to the intrinsic defects, as well as hydrogen and carbon extrinsic defects that we have also considered. The adsorption energy $\mathrm{E}_{\text {ads }}$ of $\mathrm{O}$ on $\mathrm{Se}_{\mathrm{vac}}$, referenced to atomic $\mathrm{O}$ is $\sim-7.1 \mathrm{eV}$, while that for $\mathrm{O}_{\text {ins }}$ and $\mathrm{O}_{\mathrm{ad}}$ are $-2.9 \mathrm{eV}$ and $-2.4 \mathrm{eV}$, respectively. The corresponding adsorption energies relative to molecular $\mathrm{O}_{2}$ are $-1.6 \mathrm{eV},-0.3 \mathrm{eV}$ and $0.2 \mathrm{eV}$, respectively. Here, the adsorption energy relative to molecular $\mathrm{O}_{2}$ tells us about the thermodynamic stability of the O-related defect relative to gas phase $\mathrm{O}_{2}$ in ambient conditions, and is an indication of the thermodynamic driving force of an $\mathrm{O}_{2}$ dissociation reaction. The adsorption energy relative to atomic $\mathrm{O}$, on the other hand, tells us the thermodynamic stability of the O-related defect after it has formed, in the absence of other chemical species. We have also considered $\mathrm{O}$ bound to $\mathrm{W}_{\text {vac}}$, $\mathrm{Se}$, as well as $\mathrm{O}_{2}$ bound to $\mathrm{W}_{\text {vac }}$ and $\mathrm{Se}_{\mathrm{w}}$, and in all these defects, the formation energy is at least $2.3 \mathrm{eV}$ larger (less stable). We note that our identification of the most stable O-related defects is consistent with previous literature. $^{22}$

Next, we show that kinetically, $\mathrm{O}_{2}$ can dissociate easily at $\mathrm{Se}_{\mathrm{vac}}$ sites in $\mathrm{SL}-\mathrm{WSe}$, resulting in Ose and $\mathrm{O}_{\text {ins, }}$ the most stable O-related defects. An indication of the ease of $\mathrm{O}_{2}$ dissociation at chalcogen vacancies is given by the extended $\mathrm{O}-\mathrm{O}$ bond lengths in $\mathrm{O}_{2}-\mathrm{Se}_{\mathrm{vac}}$ and $\mathrm{O}_{2}-2 \mathrm{Se}_{\mathrm{vac}}(>20 \%$ and $>90 \%$ larger than its gas phase value, respectively). Using the climbing image nudged elastic band method, ${ }^{23}$ we compute an $\mathrm{O}_{2}$ dissociation barrier of $0.52 \mathrm{eV}$ at $\mathrm{Se}_{\mathrm{vac}}$ (Fig. 2a). The reaction prefactor is computed using harmonic transition state theory, ${ }^{24}$ giving a rate constant of $\sim 10^{5}$ counts/second at $300 \mathrm{~K}$. This is consistent with the rule of thumb that a thermally activated process with barrier of $\sim 30$ $\mathrm{k}_{\mathrm{B}} \mathrm{T}(0.7 \mathrm{eV})$ or below can take place at room temperature for typical attempt frequencies. ${ }^{25}$ Zero-point energy corrections reduce the barrier height by $0.04 \mathrm{eV}$. Our 
results indicate that $\mathrm{O}_{2}$ can readily dissociate at $\mathrm{Se}_{\mathrm{vac}}$ at room temperature. In contrast, we compute a barrier of $0.94 \mathrm{eV}$ for $\mathrm{O}_{2}$ dissociation at $\mathrm{S}_{\mathrm{vac}}$ in $\mathrm{SL}-\mathrm{MoS}_{2}$, corresponding to a rate constant of only $\sim 10^{-2}$ counts/second at $300 \mathrm{~K}$. Fig. $2 \mathrm{~b}-\mathrm{c}$ shows that when $\mathrm{O}_{2}$ is adsorbed at $\mathrm{Se}_{\mathrm{vac}}$, electrons are added to the antibonding orbital of $\mathrm{O}_{2}$ and depleted from the bonding orbital and nearby $\mathrm{W}$ atoms, thus facilitating $\mathrm{O}_{2}$ dissociation. This net charge transfer to $\mathrm{O}_{2}\left(\sim 0.6 \mathrm{e}^{-} /\right.$atom $)$is in turn facilitated by the smaller work function of 2D $\mathrm{WSe}_{2}(4.69 \mathrm{eV})$ compared to $\mathrm{MoS}_{2}(5.06 \mathrm{eV})$. Based on the work functions, we expect the corresponding barriers for $\mathrm{WS}_{2}$ and $\mathrm{MoSe}_{2}$ to be larger than in $\mathrm{WSe}_{2}$ but smaller than in $\mathrm{MoS}_{2}$, a phenomenon that has also been observed in Ref. 26. Indeed, oxygen-substitutional defects have also been identified in $\mathrm{MoSe}_{2}$ and $\mathrm{WS}_{2},{ }^{20}$ while sulphur vacancies have been experimentally observed in $\mathrm{MoS}_{2}{ }^{14}$. We note that quantitative prediction of energy barriers can be challenging for DFT. However, this does not affect our conclusion that $\mathrm{O}_{2}$ can dissociate easily at $\mathrm{Se}_{\mathrm{vac}}$ sites in $\mathrm{SL}-\mathrm{WSe}$, while the same cannot be said for $\mathrm{S}_{\mathrm{vac}}$ in $\mathrm{SL}-\mathrm{MoS}_{2}$. Other exchange-correlation functionals also give a barrier of at most $0.57 \mathrm{eV}$ (see SI, Table S3). 

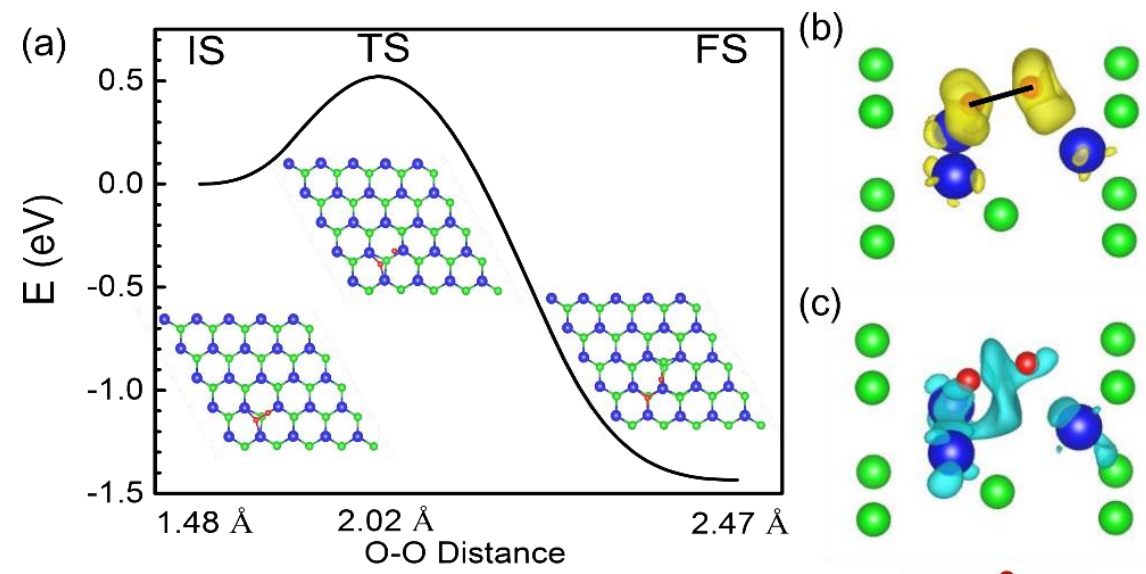

(d)

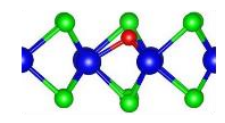

(e)

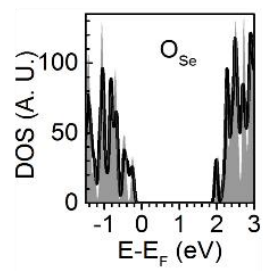

(f)

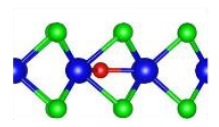

(g)

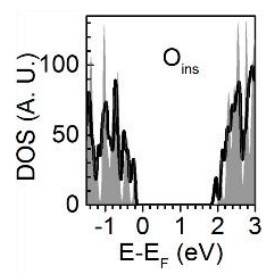

(h)

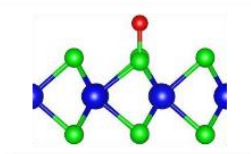

(i)

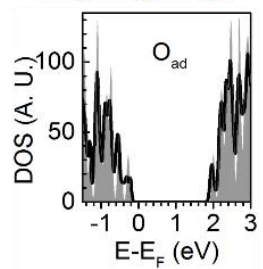

Figure 2. $\mathrm{O}_{2}$ dissociative adsorption at the Se vacancy site and O-related defects in a 5 $\times 5$ supercell. (a) $\mathrm{O}_{2}$ dissociation barrier. IS, TS and FS are initial, transition and final states, respectively. (b) Electron gain and (c) loss regions $\Delta \rho$ for the IS, computed using $\Delta \rho=\rho_{I S}-\rho_{O_{2}}-\rho_{S e_{v a c}}$. (d-i) Atomic structures and DOS (HSE06) of O-related defects. (d-e) Ose, (f-g) Oins and (h-i) Oad. Red: O, Blue: W, Green: Se. Gray shading: DOS of perfect WSe2, aligned by the 1 s core level of the $\mathrm{W}$ furthest from $\mathrm{O}$.

In the final state (Fig. 2a), one $\mathrm{O}$ atom $\mathrm{O} 1$ takes the place of the missing Se (forming $\mathrm{O}_{\mathrm{Se}}$ ), while the other, $\mathrm{O} 2$, binds to the neighbouring $\mathrm{W}$ atom. The $\mathrm{O}$ atom $\mathrm{O} 2$ can move to these more stable sites $\mathrm{O}_{\text {ins }}$ and $\mathrm{O}_{\text {ad }}$ with thermal annealing (e.g. Fig. S5). None of these defects have deep gap states (Fig. 2e, 2g, 2i). Since Sevac is the most abundant intrinsic point defect, and $\mathrm{O}_{2}$ dissociation at $\mathrm{Se}_{\mathrm{vac}}$ is facile, we expect the highly stable $\mathrm{O}_{\mathrm{Se}}$ to be the most abundant point defect in $2 \mathrm{D} \mathrm{WSe}_{2}$. 
In the above discussion, we have evaluated the possibility of $\mathrm{O}$ defects arising from $\mathrm{O}_{2}$ dissociation at chalcogen vacancies. This process is relevant to mechanically exfoliated samples, often used in optical and single photon emission experiments, as well as samples grown from elemental sources. The fact that $\mathrm{O}$ passivated Se vacancies were recently observed in 2D $\mathrm{MoSe}_{2}$ grown from elemental sources under ultra-high vacuum conditions is consistent with our theoretical predictions. ${ }^{20}$ On the other hand, CVD growth processes sometimes make use of metal oxide precursors, and it is relevant to consider the formation energy of the $\mathrm{O}$ defects under such conditions, using the chemical potential of $\mathrm{O}$ from the its reservoir $\mathrm{WO}_{3}$. Our calculations show that $\mathrm{O}_{\mathrm{se}}$ defects have the smallest formation energy (much smaller than that of $\mathrm{Se}_{\mathrm{vac}}$ ). Oins has a $\sim 1 \mathrm{eV}$ smaller formation energy than $\mathrm{Se}_{\mathrm{vac}}$ under typical Se-rich conditions (as is the case for our samples), and a comparable formation energy to $\mathrm{Se}_{\mathrm{vac}}$ under W-rich conditions (Table S1). Therefore, the $\mathrm{O}_{\mathrm{se}}$ and $\mathrm{O}_{\text {ins }}$ defects are likely to form in situ during CVD growth processes involving metal oxide precursors. We have also computed in a similar manner the formation energies of $\mathrm{O}_{\mathrm{s}}$ and $\mathrm{O}_{\text {ins }}$ defects with those of $\mathrm{S}$ vacancies in $\mathrm{MoS}_{2}$ monolayers, where we find that the Os defect is more stable than

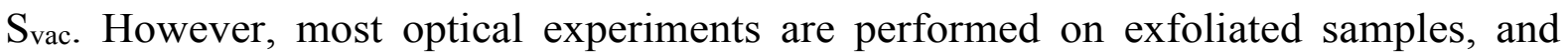
evidence of $\mathrm{S}_{\mathrm{vac}}$ was found in exfoliated $\mathrm{MoS}_{2}$ monolayers. ${ }^{1,14}$

We now present an experimental characterization of the point defects in SL-WSe2. Our low temperature STM measurements reveal the presence of three most commonly observed point defects ( $\mathrm{D}_{1}, \mathrm{D}_{2}$ and $\mathrm{D}_{3}$; Fig. 3a-c) in SL-WSe2, grown via chemical vapour deposition (CVD) on graphite ${ }^{27}$ and annealed in vacuum to $\sim 300{ }^{\circ} \mathrm{C}$. The $\mathrm{STM}$ images for $\mathrm{D}_{1}$ are similar to those in Ref. 19. STM images simulated within the TersoffHamann approximation ${ }^{28}$ for $\mathrm{O}_{\mathrm{Se}}, \mathrm{O}_{\mathrm{ins}}$ and $\mathrm{O}_{\mathrm{ad}}$ compare well with the images of $\mathrm{D}_{1}, \mathrm{D}_{2}$ and $\mathrm{D}_{3}$ (see Methods). Specifically, the detailed patterns of the defects are very different 
for different bias voltages, as revealed by the bias-dependent STM images recorded at the same area. For instance, $\mathrm{D}_{1}$ exhibits as a trefoil-like pattern at $\mathrm{V}_{\text {tip }}=1.3 \mathrm{~V}$, but shows up as a pit-like structure at $\mathrm{V}_{\text {tip }}=1.2 \mathrm{~V},-1.2 \mathrm{~V}$ and $-1.3 \mathrm{~V} ; \mathrm{D}_{2}$ exhibits three-lobe-like features without a bright central protrusion at $\mathrm{V}_{\text {tip }}=1.4 \mathrm{~V}$, but a bright central protrusion is present at $\mathrm{V}_{\text {tip }}=-1.2 \mathrm{~V}$ and $-1.3 \mathrm{~V}$. No gap states are observed in the STS spectra recorded at $\mathrm{D}_{1}-\mathrm{D}_{3}$ (Fig. 3d-f). To elucidate the origin of each defect, we first need to understand their locations by superimposing pristine $\mathrm{WSe}_{2}$ atomic structures to the STM images. It is worth noting that the bright spots in the STM images for the pristine WSe 2 can be attributed to Se sites (e.g., the $1^{\text {st }}$ row in Fig. $3 b$ and Fig. 3c) or hollow sites (the $2^{\text {nd }}$ row in Fig. 3a) depending on the bias voltages (Fig. S6). As a result, we can determine that $D_{1}$ and $D_{3}$ are located at the Se position, as shown in the $2^{\text {nd }}$ row in Fig. 3a $\left(\mathrm{V}_{\text {tip }}=1.2 \mathrm{~V}\right)$ and in Fig. $3 \mathrm{c}$, respectively, while $\mathrm{D}_{2}$ is located at the hollow site, as shown in the $1^{\text {st }}$ row in Fig. $3 \mathrm{~b}\left(\mathrm{~V}_{\text {tip }}=1.4 \mathrm{~V}\right)$. The defects $\left(\mathrm{D}_{1}\right.$ and $\left.\mathrm{D}_{3}\right)$ located at Se sites could be $\mathrm{Se}_{\mathrm{vac}}$, Ose or $\mathrm{O}_{\mathrm{ad}}$, while the one $\left(\mathrm{D}_{2}\right)$ located at the hollow site could be Oins, $\mathrm{Se}_{\text {int }}$ or $\mathrm{W}_{\text {int }}$ (Fig. S8). By comparing the simulated STM images (Figs. 3 and S8) and the experimental STM bias-dependent images, we find that $\mathrm{O}_{\mathrm{se}}, \mathrm{O}_{\text {ins }}$ and $\mathrm{O}_{\text {ad }}$ compare well with $\mathrm{D}_{1}, \mathrm{D}_{2}$ and $\mathrm{D}_{3}$ respectively.

Thus, the detailed patterns observed in the STM images for $\mathrm{D}_{1}$, which are very different for different bias voltages, strongly support the conclusion that $\mathrm{D}_{1}$ is $\mathrm{O}_{\mathrm{se}}$. The specific patterns in $\mathrm{D}_{2}$, together with the presence of $\mathrm{O}_{\mathrm{se}}$, further supports the conclusion

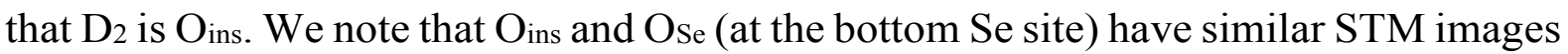
for low bias voltages. However, the STM images differ for larger bias voltages, and a comparison shows clearly that $\mathrm{D}_{2}$ corresponds to $\mathrm{O}_{\text {ins }}$ (Fig. S7). This is further supported by the different scanning tunnelling spectroscopy (STS) spectra for $\mathrm{D}_{1}$ and $\mathrm{D}_{2}$. None of the STM images simulated for the intrinsic defects (Fig. S8) match well with the 
experimental STM images for $\mathrm{D}_{1}$ and $\mathrm{D}_{2}$. Furthermore, similar to the reports in Ref. 19, no gap states are observed in the STS spectra (Fig. 3d-f). This is in contrast to the gap states predicted for intrinsic point defects and strengthens our conclusion that the defects observed here are not intrinsic point defects. Interestingly, $\mathrm{D}_{1}\left(\mathrm{O}_{\mathrm{Se}}\right)$ is observed to be the most abundant point defect, as we predict for Ose, while $\mathrm{D}_{2}$ (Oins) was also commonly observed. We were unable to locate any point defects with gap states in the STM/S experiments. This observation is consistent with the fact that the most abundant intrinsic point defect with gap states $\left(\mathrm{Se}_{\mathrm{vac}}\right.$ ) would be passivated by $\mathrm{O}$, removing the gap states, and resulting in O-related defects with no gap states. Other than $\mathrm{Se}_{\mathrm{vac}}$, the remaining intrinsic point defects with gap states have higher formation energies and are expected to occur at significantly lower densities.

(a)
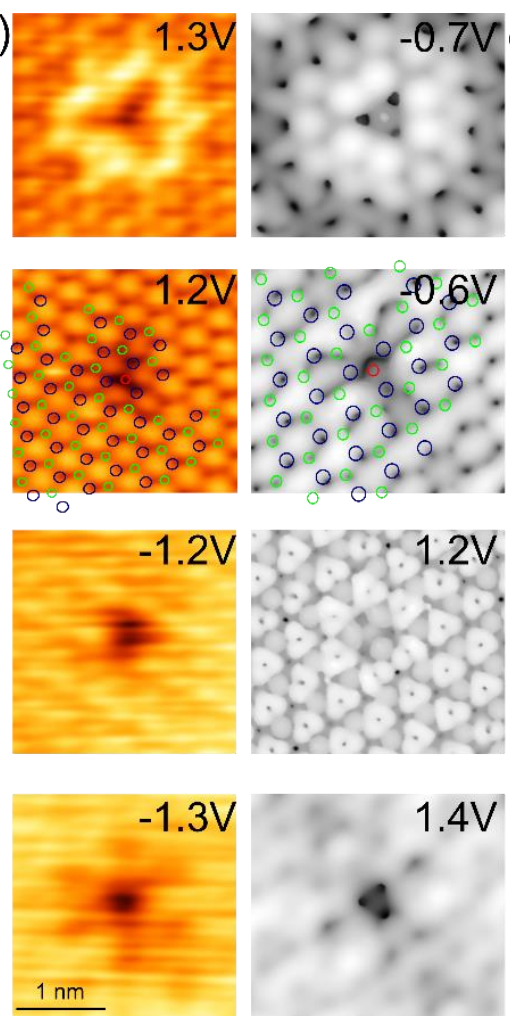

$\mathrm{D}_{1}$ vs $\mathrm{O}_{\mathrm{Se}}$
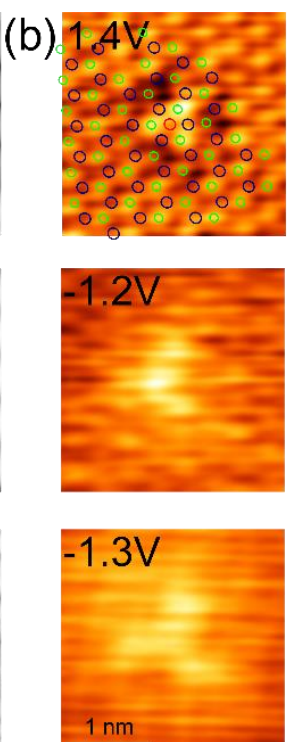

$$
\mathrm{D}_{2} \text { vs } \mathrm{O}_{\text {ins }}
$$

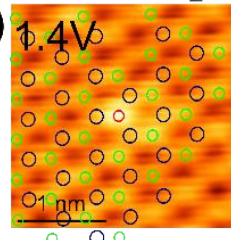

$\mathrm{D}_{3}$ vs $\mathrm{O}_{\mathrm{ad}}$
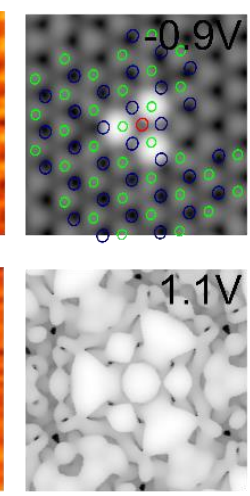

$1.2 \mathrm{~V}$
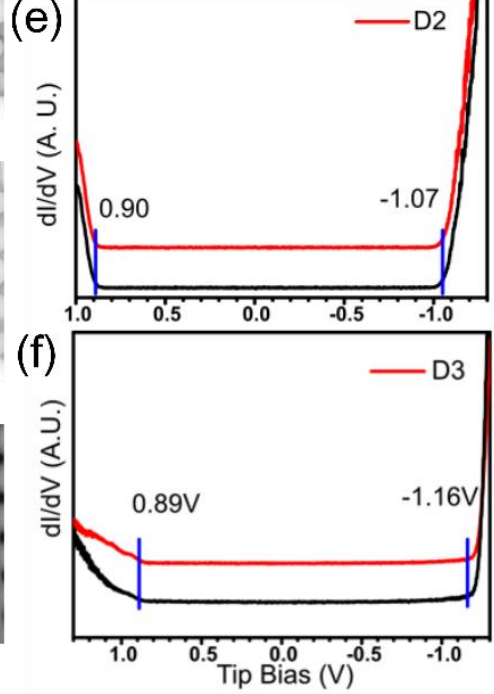
Figure 3. Experimentally observed point defects. (a-c) Left: Experimental STM images for the dominant point defects $\left(\mathrm{D}_{1}, \mathrm{D}_{2}\right.$ and $\left.\mathrm{D}_{3}\right)$ observed in STM. Right: Simulated STM images of $\mathrm{O}_{\mathrm{Se}}$, $\mathrm{O}_{\text {ins }}$ and $\mathrm{O}_{\text {ad. }}$ Voltages on the left are tip bias voltages (negative biases correspond to unoccupied states and vice versa). Voltages on the right are relative to $\mathrm{E}_{\mathrm{F}}$ in the calculation, chosen to approximate the energy ranges of states contributing to the measured STM current. Atomic positions are shown in a, b and c. (Red: O; Blue: W; Green: Se). (d-f) Averaged STS spectra on (red) and away from ( $>2 \mathrm{~nm}$, black) the defects.

STEM measurements were also performed on $\mathrm{SL}_{-W S e}$ (see Methods). Analysis of the STEM image intensities (Fig. S9) led to the conclusion that the most abundant defect observed was $\mathrm{Se}_{\text {vac}}$, while $2 \mathrm{Se}_{\text {vac }}$ and $\mathrm{Se}$ w were also present (Fig. 4). As the atomic number of $\mathrm{O}$ atoms is much smaller than that of Se and $\mathrm{W}$, and the HAADF STEM image intensity is approximately proportional to the square of the atomic number, $\mathrm{O}$ atoms are very challenging to detect above the background signal. Also, the knock-on damage threshold for the oxygen atoms may be below the $60 \mathrm{kV}$ accelerating voltage used, which can also explain why $\mathrm{O}$ was not detected. No $\mathrm{W}_{\text {vac }}$ were observed, consistent with typical Se-rich growth conditions. 


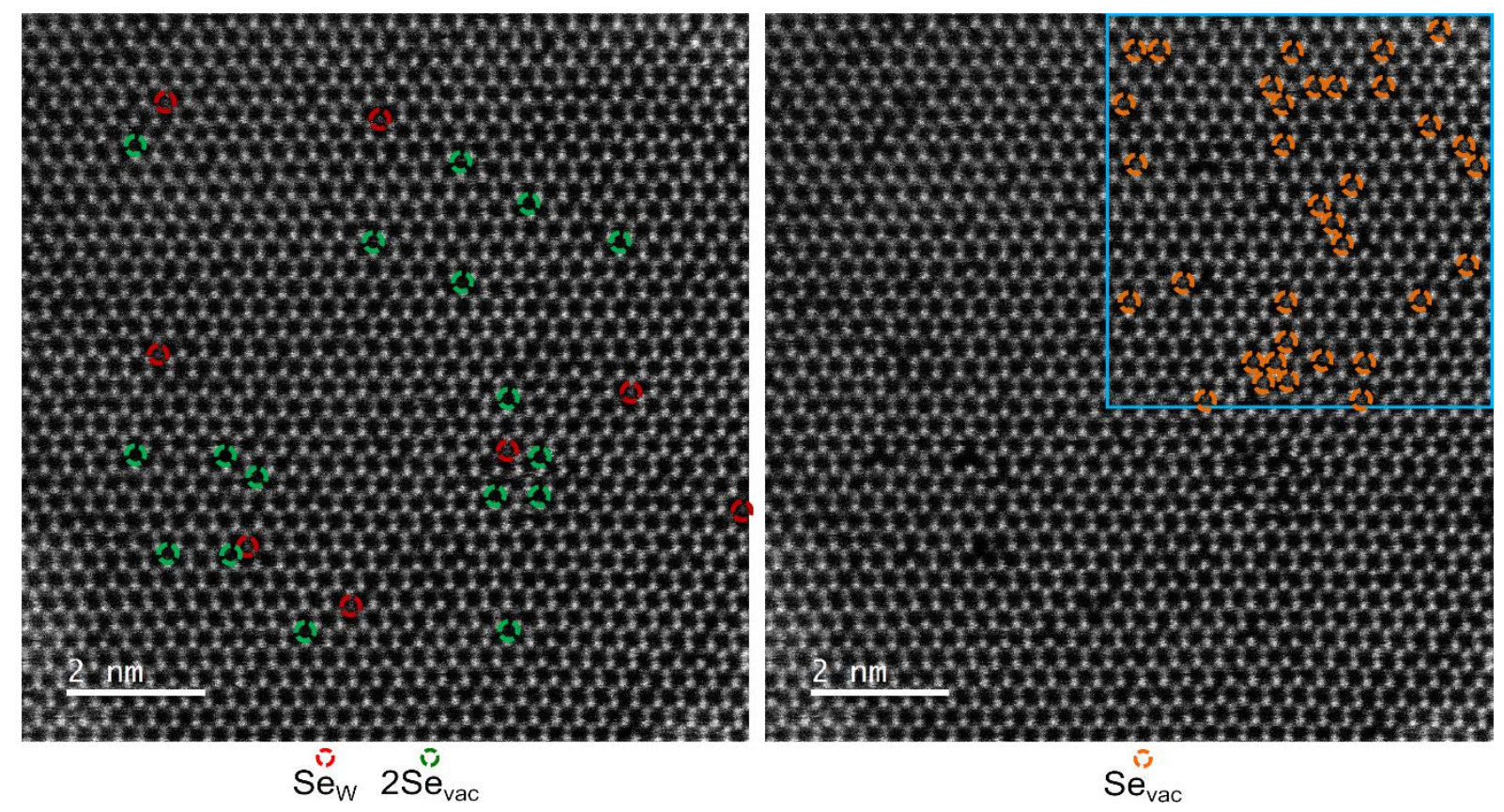

Figure 4. HAADF STEM images (60 kV accelerating voltage). The two images are for the exact same area. Sew (red) and $2 \mathrm{Se}_{\mathrm{vac}}$ (green) are marked in a, while $\mathrm{Se}_{\mathrm{vac}}$ (orange) are marked in the top right of b. O atoms cannot be distinguished due to the low knockon damage threshold and much smaller atomic number of oxygen compared to Se and W. 

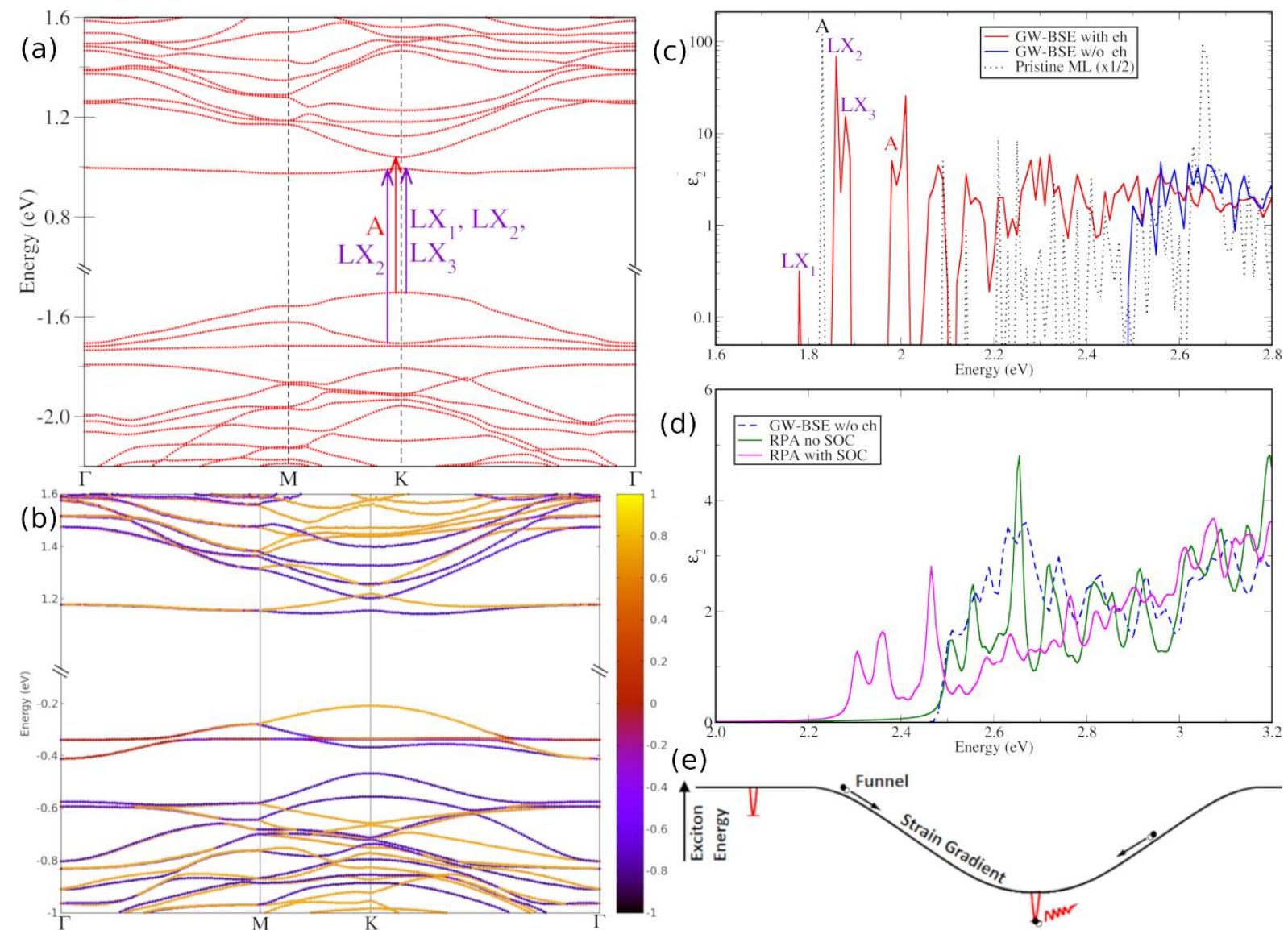

Figure 5. Electronic and optical properties for the $\mathrm{O}_{\text {ins }}$ point defect in a $4 \times 4$ supercell of SL-WSe2. (a) GW band structure, (b) DFT band structure with spin-orbit coupling (SOC) effects (the colour scale depicts the $m_{z}$ contributions for the states), (c) GW-BSE optical absorption spectrum, (d) RPA spectra with and without SOC effects, (e) Schematic showing that SPE arises from a combination of exciton funneling due to strain gradients, and exciton localization due to localized defect states such as in $\mathrm{O}_{\text {ins. }}$ Note that in (c) the broadening is reduced into $0.002 \mathrm{eV}$ with vertical axis in log-scale to emphasize the $\mathrm{LX}_{1}$ peak. The dashed line in (c) is the GW-BSE spectrum for pristine $\mathrm{SL}_{-\mathrm{WSe}}$ with no defects. The blue line represents the optical spectrum computed in the independent particle approximation (no electron-hole interactions) using the GW eigenvalues. In (d), the RPA spectrum without SOC was shifted by $+0.87 \mathrm{eV}$ to align the optical onset with the GW-BSE spectrum without electron-hole interactions (due to 
the difference in single-particle energies between the two calculations). The RPA SOC spectrum was shifted by the same amount for comparison.

We use state-of-the-art first principles GW-BSE calculations to predict the implications of these point defects on the optical response of SL-WSe2. We focus here on Ose, Oins, and Sew. The optical properties of $\mathrm{Se}_{\mathrm{vac}}$ and $\mathrm{W}_{\mathrm{vac}}$ have been studied in the literature. ${ }^{17}$ The GW-BSE calculations account for electron self-energy effects in the quasiparticle spectra as well as electron-hole interactions in the excitons. State-of-theart non-uniform k-point interpolation methods ${ }^{18,29}$ are used to obtain good convergence with respect to k-point sampling. $4 \times 4$ supercells are considered for these calculations, while optical properties are also computed in the random phase approximation (RPA) for $7 \times 7$ supercells as a comparison. Fully relativistic RPA calculations as well as GWBSE calculations with spin-orbit coupling (SOC) added perturbatively, are also performed for $4 \times 4$ cells to estimate the effects of SOC on the optical spectra. We remark that our focus in this manuscript is on the low-energy excitons, with energies comparable to or smaller than that of the free exciton A peak. For these excitons, we expect our optical spectra to be converged.

We first focus on the Oins defect (Fig. 5). Interestingly, there is a low energy exciton $\left(\mathrm{LX}_{1}\right.$; Fig. 5c) at $1.78 \mathrm{eV}, 50 \mathrm{meV}$ below the energy of the free exciton A peak in pristine $\mathrm{WSe}_{2}$. Analysis of the exciton wavefunctions (Table S5) shows that the A peak in the defect cell is at $1.97 \mathrm{eV}$ (Fig. 5c), and that $\mathrm{LX}_{2}$ and $\mathrm{LX}_{3}$ are also defect-related excitons $30 \mathrm{meV}$ and $50 \mathrm{meV}$ above the energy of the A peak in pristine $\mathrm{WSe}_{2}$, respectively. The difference in energy between $\mathrm{LX}_{1}$ and the A peak lies in the same range as that experimentally observed in SL-WSe $2(\sim 45-100 \mathrm{meV})$, where the A peak in pristine $\mathrm{WSe}_{2}$ is relevant in the limit of low defect densities. ${ }^{5-10}$ 
We note here that not all localized excitons are going to give rise to SPE. Instead, SPE is widely believed to arise from localized defects that are located in regions with tensile strain (which reduces the quasiparticle gap), funneling the electron and hole pair to the region of the defect ${ }^{4,30}$ (Fig. 5e). Experimentally, it has been found that $\sim 2.1 \%$ applied strain red-shifts the exciton peak by $0.1 \mathrm{eV}$ in SL-WSe2. ${ }^{31}$ The slight red shift of the exciton peak with strain is also consistent with recent calculations. ${ }^{32} \mathrm{We}$ expect that for the samples that have not been strained deliberately, ${ }^{5-10}$ similar amounts of strain would be present in the region of the quantum emitters, red-shifting the localized exciton peaks by $\sim 100 \mathrm{meV}$, compared to the pristine case. This red-shift does not change our conclusions that the LX excitons in $\mathrm{O}_{\text {ins }}$ lie in the energy range of experimentally observed single photons. ${ }^{5-10}$ In particular, as we discuss below, no other point defects considered here give similar spectra.

For single photon emission, it is important that the exciton is localized. Indeed, we see that $\mathrm{LX}_{1}$ primarily results from a combination of the bulk VBM state and defect CBM state at the K point (Fig. 5a; Table S5; Fig. S10), while other LX excitons are related to transitions between the VBM/VBM-1 states and defect CBM state. The localized nature of the defect state supports the emission of single photons, while the involvement of the bulk state is consistent with the valley polarization ${ }^{18}$ of single photons observed in Ref. 5. From Fig. 5c, we note that electron-hole interactions are important for reducing the optical onset and creating separate energy peaks (LX versus A), pointing to the importance of excitonic effects in this defect system.

We further remark that screening from the substrates should not have a significant effect on the exciton peak positions. In general, screening from the substrates reduces the quasiparticle gap and the exciton binding energy by a similar amount. Since the optical gap is the difference between the quasiparticle gap and exciton binding energy, 18 
the effect of substrates on the optical peak positions is much smaller, as shown previously in $\mathrm{MoSe}_{2}{ }^{33}$ and $\mathrm{MoS}_{2}{ }^{34}$ Furthermore, the fact that different substrates $\left(\mathrm{SiO}_{2} / \mathrm{Si}^{5-7,9}\right.$ hexagonal boron nitride/graphene $\left.{ }^{8,10}\right)$ give similar SPE peak positions also shows that the effect of substrate on the SPE peak positions is negligible.
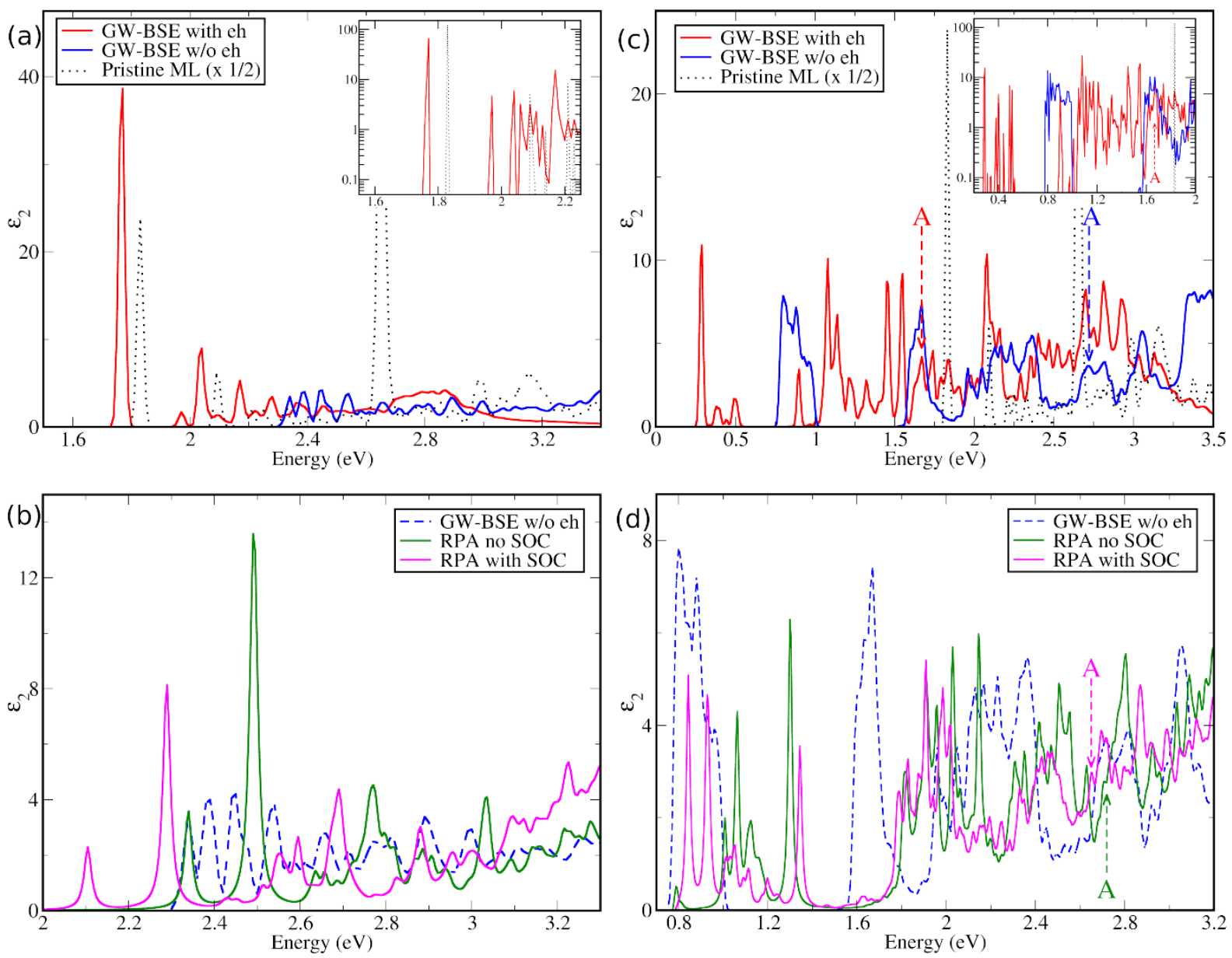

Figure 6. Optical absorption spectra for Ose and Sew point defects, each in a $4 \times 4$ supercell of SL-WSe2. (a) GW-BSE and (b) RPA spectra for Ose, (c) GW-BSE and (d) RPA spectra for Sew. The RPA spectra in (b) and (d) are shifted by +0.92 and $+0.77 \mathrm{eV}$, respectively, to match the GW-BSE spectra without electron-hole interactions. Labels A in (c) and (d) mark the free exciton A peak energy positions in the respective spectra. Insets in (a) and (c) show the low energy GW-BSE spectra plotted with a log-scale and smaller broadening. 
In Fig. 6, we show the optical absorption spectra computed for OSe and Sew defects. For the Ose defect system, there are no lower energy excitons below the first prominent peak, which corresponds to the free exciton A peak and is not a localized exciton. The optical band gap is narrowed to $1.76 \mathrm{eV}, 60 \mathrm{meV}$ lower than that of pristine $\mathrm{SL}-\mathrm{WSe}$. For the Sew defect, the deep defect states (Fig. 1c) result in a large number of bound excitonic transition levels below the A peak, spanning a wide energy range $(\sim 1.56 \mathrm{eV})$, down to $\sim 0.3 \mathrm{eV}$ (Fig. 6c). This implies that Sew defects cannot be responsible for the SPE previously observed in SL-WSe2. However, we predict that Sew point defects are promising for SPE in the infrared and near-infrared spectral range.

We now consider the effect of spin orbit coupling (SOC) on our conclusions. The DFT band structures of the defect supercells, including SOC effects, are shown in Fig. $5 \mathrm{~b}$ and Fig. S11-12. We first estimate the effects of SOC by comparing DFT RPA spectra (independent particle approximation) with and without SOC (Fig. 5d, 6b, 6d). There is no obvious change in the optical activity of the transitions and the two spectra are in general very similar, except for a narrowing of the optical onset by $\sim 0.2 \mathrm{eV}$ because of the smaller DFT band gap when SOC is included. To quantify the effect of SOC on the difference in energy between $\mathrm{LX}_{1}$ and the A peak in the pristine cell, we include the effect of SOC perturbatively in our GW-BSE calculations. ${ }^{35}$ We note that the $\mathrm{m}_{\mathrm{z}}$ projection values for the $\mathrm{O}_{\text {ins }}$ band structure near the band edges (as shown in Fig. $5 b)$ are still large at $\sim 0.75$. These projection values are similar to that in pristine $\mathrm{WSe}_{2}$ (Fig. S17a), where full spinor GW-BSE calculations and GW-BSE calculations without SOC yield comparable oscillator strengths for the A peak (Fig. S17c). A fully converged GW-BSE calculation with the perturbative SOC approach results in an A peak at $1.6 \mathrm{eV}$ and a $\mathrm{B}$ peak at $2.0 \mathrm{eV}$ in pristine $\mathrm{SL}-\mathrm{WSe}_{2}$, close to the experimentally measured 1.75 $\mathrm{eV}$ and $2.20 \mathrm{eV}$ (Fig. S17b). ${ }^{36}$ With the perturbative SOC procedure, the $\mathrm{LX}_{1}$ peak is 16 
meV above the A peak in pristine SL-WSe2. Together with the effect of strain on the SPE as discussed above, we obtain an $\mathrm{LX}_{1}$ peak position of $\sim 84 \mathrm{meV}$ below the pristine A peak in this approximation. The GW-BSE spectra with SOC included perturbatively are shown in Fig. S13 for $\mathrm{O}_{\text {ins, }} \mathrm{O}_{\mathrm{Se}}$, and Sew defects. Even with the inclusion of SOC, Oins is much more likely than any other defect to be responsible for SPE within the energy range previously detected in experiment. The fact that $\mathrm{O}_{\text {ins }}$ has found to exist in significant concentrations compared to other point defects with localized excitons further strengthens our conclusion.

In Fig. S14, we compare the RPA spectra for $4 \times 4$ and $7 \times 7$ supercells. For $O_{\text {ins }}$ and $\mathrm{OSe}_{\mathrm{Se}}$, the RPA spectra for $4 \times 4$ and $7 \times 7$ supercells are very similar, apart from a small shift in energy. For Sew, the $7 \times 7$ supercell has fewer peaks in the RPA spectrum, but these peaks are still deep in the band gap and thus do not change our qualitative results that Sew is likely to emit in the infrared range. From Fig. S16, we also see that the DFT PBE band structures of the $\mathrm{O}_{\text {ins }}$ system are very similar for $4 \times 4$ and $7 \times 7$ supercells.

\section{CONCLUSIONS}

We have presented a detailed study to identify the point defects in SL-WSe2, and to predict, using state-of-the-art calculations, the nature and energies of excitons at these defect sites. Our DFT and STM results show that $\mathrm{O}_{\mathrm{Se}}$ is the most abundant defect in 2D $\mathrm{WSe}_{2}$, passivating the gap states. This explains the superior optical quality of $\mathrm{WSe}_{2}$, and facilitates the observation of SPE from localized excitons. O-substituted chalcogen vacancies have also been observed in $\mathrm{MoSe}_{2}$ and $\mathrm{WS}_{2},{ }^{20}$ but not in mechanically exfoliated $\mathrm{MoS}_{2}$, where $\mathrm{S}$ vacancies with no $\mathrm{O}$ have been observed. ${ }^{14}$ These findings are also consistent with our theoretical predictions and contribute to a unified picture of 
the interaction of oxygen with chalcogen vacancies in TMDs. We further identify $\mathrm{O}_{\text {ins }}$ and Sew to be point defects present in $2 \mathrm{D} \mathrm{WSe} 2$.

The identity of point defects in 2D materials is important for many applications. In this work, we have predicted the implications of the experimentally observed point defects on the optical response. Our calculations are motivated by the SPE experiments reported in $\mathrm{SL}_{-} \mathrm{WSe}_{2},{ }^{5-10}$ and also by the fact that it is currently challenging experimentally to ascertain unambiguously the nature of the point defect responsible for SPE, thus underscoring the importance of first principles calculations to provide key insights and guide experimentalists in future studies. The GW-BSE calculations employed here are state-of-the-art, converged with the best available k-point interpolation methods, ${ }^{29}$ and comparable in quality with recent high quality GW-BSE calculations for defects in $2 \mathrm{D}$ materials. ${ }^{18} \mathrm{We}$ have also discussed the implications of strain, supercell size, spin-orbit coupling and substrate screening on our conclusions. The key point here is that of all the experimentally observed point defects, only one candidate $\left(\mathrm{O}_{\text {ins }}\right)$ is available that gives localized excitons in the energy range observed in recent single photon emission experiments. ${ }^{5-10}$ Small numerical shifts that arise from strain or other effects do not change this conclusion. Our conclusion is also consistent with the fact that only Oins has a flat defect band close to the band edge of WSe2, in contrast to other candidate point defects, which have defect states deep in the band gap, thus giving rise to photon energies that are much different from that observed in the experiments. ${ }^{5-10}$ We emphasize that based on our results, the previous observations ${ }^{5-10}$ of single photon emission from $\mathrm{WSe}_{2}$ are related to the superior optical quality of $\mathrm{WSe}_{2}$ due to the passivation of gap states by $\mathrm{O}$, the presence of a flat $\mathrm{O}_{\text {ins }}$ defect band close to the $\mathrm{WSe}_{2}$ band edges, as well as the presence of strain gradients for exciton funneling. Low-lying dark excitons could also help increase the lifetime of excitons to facilitate 
the funneling process. These predictions suggest ways to create quantum emitters in other semiconducting TMDs, e.g. through controlled reaction with $\mathrm{O}_{2} .^{22}$ Recently, single-photon emission has been observed in encapsulated 2D $\mathrm{MoS}_{2}$, following helium ion bombardment. ${ }^{37}$ In this case, encapsulation removes the effects of contaminants on sulphur vacancies and other defects, while helium bombardment is believed to create Mo vacancies with localized defect states. Indeed, for single photon emission, it is not necessary for defect states to be close to the band edges, because single photons of other energies can also be considered. Our predictions also point experimentalists toward other energy ranges in which single photons may be emitted in the TMDs.

\section{MATERIALS AND METHODS}

Except for calculations on optical properties, all our DFT calculations are performed using the VASP $\operatorname{code}^{38,39}$ with the PAW approach. We use the PBE exchange-correlation functional ${ }^{40}$ with Grimme's D2 ${ }^{41}$ correction for van der Waals $(\mathrm{vdW})$ interactions for all calculations except the density of states (DOS) plots which were performed with the hybrid $\mathrm{HSE}^{42} 6^{42}$ exchange-correlation functional. Spin polarization is included for the defect structures and for oxygen.

STM images were simulated in the Tersoff-Hamann approximation. ${ }^{28}$ The TersoffHamann approximation predicts STM images ${ }^{14}$ for $\mathrm{S}_{\mathrm{vac}}$ in $\mathrm{SL}-\mathrm{MoS}_{2}$ in good agreement with experiment, and with more sophisticated calculations including tip effects. ${ }^{43}$ Besides the images obtained with the PBE exchange-correlation functional (Fig. 3), we have also simulated images using the $\mathrm{HSE}^{42} 6^{42}$ functional (Fig. S15), which also agree well with experiment. 
Our GW-BSE calculations are performed with Quantum-ESPRESSO ${ }^{44}$ and BerkeleyGW ${ }^{45}$ software. PBE optimized norm-conserving Vanderbilt (ONCV) pseudopotentials ${ }^{46}$ were used, with a kinetic energy cutoff of $60 \mathrm{Ry}$. W semicore $5 \mathrm{~s}, 5 \mathrm{p}$ and $5 \mathrm{~d}$ states were included as valence electrons. Each defect is simulated in a $4 \times 4$ supercell, with atomic positions fully relaxed and with the lattice parameters fixed at 4 times of the primitive $\mathrm{WSe}_{2}$ cell. We performed one-shot $\mathrm{G}_{0} \mathrm{~W}_{0}$ calculations with a dielectric matrix cutoff of $15 \mathrm{Ry}$, slab Coulomb truncation, ${ }^{47}$ the Hybertsen-Louie generalized plasmon pole model, ${ }^{48}$ and the static remainder method to speed up convergence. ${ }^{49}$ The reciprocal space was sampled using non-uniform neck subsampling (NNS) in GW and cluster sampling interpolation (CSI) in BSE ${ }^{29}$ Using these methods, we converged the GW quasiparticle energies in the primitive WSe2 cell with 520 bands and using a $15 \times 15 \times 1 \mathrm{q}$-mesh with an additional $10 \mathrm{q}$-points in the Voronoi cell around $\mathrm{q}=0$, which is equivalent to an effective uniform grid of more than $150 \times 150 \times 1$ sampling. The GW gap at the $\mathrm{K}$ point is computed to be $2.342 \mathrm{eV}$. Keeping all parameters the same and reducing the number of bands to 92, we obtain a GW gap at K of $2.367 \mathrm{eV}$. We converged the BSE spectrum using a $24 \times 24 \times 1$ coarse mesh interpolated onto a $72 \times 72 \times 1$ fine mesh and the CSI scheme with sub-factor 3 , which translates to an additional $10 \mathrm{k}$-points sampled of kernel elements for each cluster along the (110) direction. We checked that the spectrum is converged within $0.01 \mathrm{eV}$ against a $90 \times 90$ $\times 1$ fine mesh calculation. In the $4 \times 4$ supercells with defects, we used a $4 \times 4 \times 1$ qmesh with 10 q-point NNS, and 1500 bands to compute the GW eigenvalues, and for the BSE part, we used a $6 \times 6 \times 1 \mathrm{k}$-mesh interpolated onto a $18 \times 18 \times 1$ fine mesh, together with the CSI method using sub-factor 3 for an additional $10 \mathrm{k}$-points sampled for each cluster along (110). The supercell k-mesh is converged at the same level as the 
primitive cell. The BSE equation is solved by direct diagonalization within the TammDancoff apporoximation (TDA), including bands with energy at least $0.5 \mathrm{eV}$ beyond the VBM and CBM. The perturbative treatment of spin-orbit coupling in GW-BSE optical spectra follow the procedure in Ref. 35.

RPA calculations as well as GW-BSE with full spinor wavefunctions were performed with Yambo. ${ }^{50}$

STEM measurements were performed on SL-WSe 2, CVD grown on sapphire ${ }^{27}$ and transferred to a TEM grid for measurement. A $60 \mathrm{kV}$ accelerating voltage was used.

Further details on methods used are provided in the SI.

\section{ASSOCIATED CONTENT}

\section{Supporting Information:}

Further details on Methods, STM images of all intrinsic defects, STEM analysis, Exciton wavefunction analysis, GW-BSE spectra with SOC, RPA spectra for $7 \times 7$ supercells, and other information. This material is available free of charge via the Internet at http://pubs.acs.org.

\section{AUTHOR INFORMATION}

\section{Corresponding authors:}

*E-mail: $\quad$ phyqsy@nus.edu.sg (theory); phyweets@nus.edu.sg (STM); msepsj@nus.edu.sg (STEM).

$\dagger$ Yu Jie Zheng and Yifeng Chen contributed equally. 


\section{NOTES}

Conflict of interests: The authors declare no competing financial interests.

\section{ACKNOWLEDGEMENTS}

SYQ acknowledges support from grant NRF-NRFF2013-07 from the National Research Foundation (NRF), Singapore. AW, YLH and PKG acknowledge support from ASTAR Pharos grant R-144-000-359-305. SYQ, SJP and AW acknowledge support from the Singapore NRF, Prime Minister's Office, under its medium-sized centre program. SJP is grateful to the National University of Singapore for support. SJP and PKG acknowledge MOE grant number R-144-000-389-114. Computations were performed on the CA2DM cluster and the National Supercomputing Centre (NSCC) in Singapore. YJZ acknowledges an NUS research scholarship, and discussions with Zijing Ding and Zhibo Song. We thank S. Refaely-Abramson for technical advice on using the NNS and CSI methods, and G. Eda for discussions.

\section{REFERENCES}

1. Amani, M.; Lien, D. H.; Kiriya, D.; Xiao, J.; Azcatl, A.; Noh, J.; Madhvapathy, S. R.; Addou, R.; Santosh, K. C.; Dubey, M.; Cho, K.; Wallace, R. M.; Lee, S. C.; He, J. H.; Ager, J. W.; Zhang, X.; Yablonovitch, E.; Javey, A., Near-Unity Photoluminescence Quantum Yield in $\mathrm{MoS}_{2}$. Science 2015, 350, 1065-1068.

2. Garcia, J. H.; Vila, M.; Cummings, A. W.; Roche, S., Spin Transport in Graphene/Transition Metal Dichalcogenide Heterostructures. Chem. Soc. Rev. 2018, 47, 3359-3379.

3. Hogele, A.; Galland, C.; Winger, M.; Imamoglu, A., Photon Antibunching in the Photoluminescence Spectra of a Single Carbon Nanotube. Phys. Rev. Lett. 2008, 100, 217401.

4. Branny, A.; Kumar, S.; Proux, R.; Gerardot, B. D., Deterministic Strain-Induced Arrays of Quantum Emitters in a Two-Dmensional Semiconductor. Nat. Commun. 2017, $8,15053$. 
5. Srivastava, A.; Sidler, M.; Allain, A. V.; Lembke, D. S.; Kis, A.; Imamoglu, A., Optically Active Quantum Dots in Monolayer WSe2. Nat. Nanotechnol. 2015, 10, 491496.

6. He, Y. M.; Clark, G.; Schaibley, J. R.; He, Y.; Chen, M. C.; Wei, Y. J.; Ding, X.; Zhang, Q.; Yao, W.; Xu, X. D.; Lu, C. Y.; Pan, J. W., Single Quantum Emitters in Monolayer Semiconductors. Nat. Nanotechnol. 2015, 10, 497-502.

7. Chakraborty, C.; Kinnischtzke, L.; Goodfellow, K. M.; Beams, R.; Vamivakas, A. N., Voltage-Controlled Quantum Light from an Atomically Thin Semiconductor. Nat. Nanotechnol. 2015, 10, 507-512.

8. Clark, G.; Schaibley, J. R.; Ross, J.; Taniguchi, T.; Watanabe, K.; Hendrickson, J. R.; Mou, S.; Yao, W.; Xu, X. D., Single Defect Light-Emitting Diode in a van der Waals Heterostructure. Nano Lett. 2016, 16, 3944-3948.

9. Koperski, M.; Nogajewski, K.; Arora, A.; Cherkez, V.; Mallet, P.; Veuillen, J. Y.; Marcus, J.; Kossacki, P.; Potemski, M., Single Photon Emitters in Exfoliated WSe2 Structures. Nat. Nanotechnol. 2015, 10, 503-506.

10. Schwarz, S.; Kozikov, A.; Withers, F.; Maguire, J. K.; Foster, A. P.; Dufferwiel, S.; Hague, L.; Makhonin, M. N.; Wilson, L. R.; Geim, A. K.; Novoselov, K. S.; Tartakovskii, A. I., Electrically Pumped Single-Defect Light Emitters in WSe $2.2 D$ Mater. 2016, 3, 025038.

11. Deilmann, T.; Thygesen, K. S., Dark Excitations in Monolayer Transition Metal Dichalcogenides. Phys. Rev. B 2017, 96, 201113.

12. Echeverry, J. P.; Urbaszek, B.; Amand, T.; Marie, X.; Gerber, I. C., Splitting Between Bright and Dark Excitons in Transition Metal Dichalcogenide Monolayers. Phys. Rev. B 2016, 93, 121107.

13. Ross, J. S.; Klement, P.; Jones, A. M.; Ghimire, N. J.; Yan, J. Q.; Mandrus, D. G.; Taniguchi, T.; Watanabe, K.; Kitamura, K.; Yao, W.; Cobden, D. H.; Xu, X. D., Electrically Tunable Excitonic Light-Emitting Diodes Based on Monolayer WSe $2 P-N$ Junctions. Nat. Nanotechnol. 2014, 9, 268-272.

14. Vancso, P.; Magda, G. Z.; Peto, J.; Noh, J. Y.; Kim, Y. S.; Hwang, C.; Biro, L. P.; Tapaszto, L., The Intrinsic Defect Structure of Exfoliated $\mathrm{MoS}_{2}$ Single Layers Revealed by Scanning Tunneling Microscopy. Sci. Rep. 2016, 6, 29726.

15. Haldar, S.; Vovusha, H.; Yadav, M. K.; Eriksson, O.; Sanyal, B., Systematic Study of Structural, Electronic, and Optical Properties of Atomic-Scale Defects in the Two-Dimensional Transition Metal Dichalcogenides $\mathrm{MX}_{2}(\mathrm{M}=\mathrm{Mo}, \mathrm{W} ; \mathrm{X}=\mathrm{S}, \mathrm{Se}, \mathrm{Te})$. Phys. Rev. B 2015, 92, 235408.

16. Moody, G.; Tran, K.; Lu, X. B.; Autry, T.; Fraser, J. M.; Mirin, R. P.; Yang, L.; Li, X. Q.; Silverman, K. L., Microsecond Valley Lifetime of Defect-Bound Excitons in Monolayer WSe2. Phys. Rev. Lett. 2018, 121, 057403.

17. Jiang, J.; Pachter, R.; Mou, S., Tunability in the Optical Response of Defective Monolayer WSe2 by Computational Analysis. Nanoscale 2018, 10, 13751-13760.

18. Refaely-Abramson, S.; Qiu, D. Y.; Louie, S. G.; Neaton, J. B., Defect-Induced Modification of Low-Lying Excitons and Valley Selectivity in Monolayer Transition Metal Dichalcogenides. Phys. Rev. Lett. 2018, 121, 167402.

19. Zhang, S.; Wang, C.-G.; Li, M.-Y.; Huang, D.; Li, L.-J.; Ji, W.; Wu, S., Defect Structure of Localized Excitons in a WSe 2 Monolayer. Phys. Rev. Lett. 2017, 119, 046101.

20. Barja, S.; Refaely-Abramson, S.; Schuler, B.; Qiu, D. Y.; Pulkin, A.; Wickenburg, S.; Ryu, H.; Uega, M. M.; Kastl, C.; Chen, C.; Hwang, C.; Schwartzberg, A.; Aloni, S.; Mo, S.-K.; Ogletree, D. F.; Cromme, M. F.; Yazyev, O. V.; Louie, S. G.; Neaton, J. B.; Weber-Bargioni, A., Identifying Substitutional Oxygen as a Prolific Point Defect in 
Monolayer Transition Metal Dichalcogenides with Experiment and Theory. 2018, arXiv:1810.03364. arXiv.org e-Print archive. https://arxiv.org/abs/1810.03364 (accessed May 8, 2019).

21. Zheng, Y. J.; Chen, Y.; Huang, Y. L.; Gogoi, P. K.; Li, M. Y.; Li, L.-J.; Trevisanutto, P. E.; Wang, Q.; Pennycook, S. J.; Wee, A. T. S., The Origin of Single Photon Emission in 2D WSe2. 2018, arXiv: 1811.00221. arXiv.org e-Print archive. https://arxiv.org/abs/1811.00221 (accessed May 8, 2019).

22. Lu, J. P.; Carvalho, A.; Chan, X. K.; Liu, H. W.; Liu, B.; Tok, E. S.; Loh, K. P.; Neto, A. H. C.; Sow, C. H., Atomic Healing of Defects in Transition Metal Dichalcogenides. Nano Lett. 2015, 15, 3524-3532.

23. Henkelman, G.; Uberuaga, B. P.; Jonsson, H., A Climbing Image Nudged Elastic Band Method for Finding Saddle Points and Minimum Energy Paths. J. Chem. Phys. 2000, 113, 9901-9904.

24. Henkelman, G. Methods for Calculating Rates of Transitions with Application to Catalysis and Crystal Growth. Ph.D. Thesis, University of Washington, 2001, Chapter 4, Pages 60-69.

25. Zhu, T.; Li, J.; Yip, S., Atomistic Reaction Pathway Sampling: the Nudged Elastic Band Method and Nanomechanics Applications. In Nano and Cell Mechanics, Espinosa, H. D.; Bao, G., Eds. John Wiley \& Sons, Ltd: 2013; pp 311-338.

26. Liu, H. S.; Han, N. N.; Zhao, J. J., Atomistic Insight into the Oxidation of Monolayer Transition Metal Dichalcogenides: from Structures to Electronic Properties. RSC Adv. 2015, 5, 17572-17581.

27. Huang, J. K.; Pu, J.; Hsu, C. L.; Chiu, M. H.; Juang, Z. Y.; Chang, Y. H.; Chang, W. H.; Iwasa, Y.; Takenobu, T.; Li, L. J., Large-Area Synthesis of Highly Crystalline $\mathrm{WSe}_{2}$ Mono layers and Device Applications. ACS Nano 2014, 8, 923-930.

28. Tersoff, J.; Hamann, D. R., Theory of The Scanning Tunneling Microscope. Phys. Rev. B 1985, 31, 805-813.

29. da Jornada, F. H.; Qiu, D. Y.; Louie, S. G., Nonuniform Sampling Schemes of The Brillouin Zone for Many-Electron Perturbation-Theory Calculations in Reduced Dimensionality. Phys. Rev. B 2017, 95, 03109.

30. Feng, J.; Qian, X.; Huang, C.-W.; Li, J., Strain-Engineered Artificial Atom as a Broad-Spectrum Solar Energy Funnel. Nat. Photonics 2012, 6, 866.

31. Aslan, O. B.; Deng, M. D.; Heinz, T. F., Strain Tuning of Excitons in Monolayer WSe2. Phys. Rev. B 2018, 98, 115308.

32. Feierabend, M.; Morlet, A.; Berghäuser, G.; Malic, E., Impact of Strain on the Optical Fingerprint of Monolayer Transition-Metal Dichalcogenides. Phys. Rev. B 2017, 96, 045425.

33. Ugeda, M. M.; Bradley, A. J.; Shi, S. F.; da Jornada, F. H.; Zhang, Y.; Qi, D. C.; Ruan, W.; Mo, S. K.; Hussain, Z.; Shen, Z.-X.; Wang, F.; Louie, S. G.; Cromme, M. F., Giant Bandgap Renormalization and Excitonic Effects in a Monolayer Transition Metal Dichalcogenide Semiconductor. Nat. Mater. 2014, 13, 1091.

34. Klots, A. R.; Newaz, A. K. M.; Wang, B.; Prasai, D.; Krzyzanowska, H.; Lin, J. H.; Caudel, D.; Ghimire, N. J.; Yan, J.; Ivanov, B. L.; Velizhanin, K. A.; Burger, A.; Mandrus, D. G.; Tolk, N. H.; Pantelides, S. T.; Bolotin, K. I., Probing Excitonic States in Suspended Two-Dimensional Semiconductors by Photocurrent Spectroscopy. Sci. Rep. 2014, 4, 6608.

35. Qiu, D. Y.; da Jornada, F. H.; Louie, S. G., Screening and Many-Body Effects in Two-Dimensional Crystals: Monolayer MoS 2 . Phys. Rev. B 2016, 93, 235435. 
36. Arora, A.; Koperski, M.; Nogajewski, K.; Marcus, J.; Faugeras, C.; Potemski, M., Excitonic Resonances in Thin Films of WSe2: from Monolayer to Bulk Material. Nanoscale 2015, 7, 10421-10429.

37. Klein, J.; Lorke, M.; Florian, M.; Sigger, F.; Wierzbowski, J.; Cerne, J.; Muller, K.; Taniguchi, T.; Watanabe, K.; Wurstbauer, U.; Kaniber, M.; Knap, M.; Schmidt, R.; Finley, J. J.; Holleitner, A. W., Atomistic Defect States as Quantum Emitters in Monolayer $\mathrm{MoS}_{2}$. 2019, arXiv: 1901.01042. arXiv.org e-Print archive. https://arxiv.org/abs/1901.01042 (accessed May 8, 2019).

38. Kresse, G.; Furthmüller, J., Efficient Iterative Schemes for Ab Initio TotalEnergy Calculations Using a Plane-Wave Basis Set. Phys. Rev. B 1996, 54, 1116911186.

39. Kresse, G.; Furthmüller, J., Efficiency of Ab-Initio Total Energy Calculations for Metals and Semiconductors Using a Plane-Wave Basis Set. Comp. Mater. Sci. 1996, 6, 15-50.

40. Perdew, J. P.; Burke, K.; Ernzerhof, M., Generalized Gradient Approximation Made Simple. Phys. Rev. Lett. 1996, 77, 3865-3868.

41. Grimme, S., Semiempirical GGA-Type Density Functional Constructed with a Long-Range Dispersion Correction. J. Comput. Chem. 2006, 27, 1787-1799.

42. Krukau, A. V.; Vydrov, O. A.; Izmaylov, A. F.; Scuseria, G. E., Influence of the Exchange Screening Parameter on the Performance of Screened Hybrid Functionals. $J$. Chem. Phys. 2006, 125, 224106.

43. González, C.; Biel, B.; Dappe, Y., Theoretical Characterisation of Point Defects on a $\mathrm{MoS}_{2}$ Monolayer by Scanning Tunnelling Microscopy. Nanotechnology 2016, 27, 105702 .

44. Giannozzi, P.; Baroni, S.; Bonini, N.; Calandra, M.; Car, R.; Cavazzoni, C.; Ceresoli, D.; Chiarotti, G. L.; Cococcioni, M.; Dabo, I.; Dal Corso, A.; de Gironcoli, S.; Fabris, S.; Fratesi, G.; Gebauer, R.; Gerstmann, U.; C., G.; Kokalj, A.; Lazzeri, M.; Martin-Samos, L., et al., QUANTUM ESPRESSO: a Modular and Open-Source Software Project for Quantum Simulations of Materials. J. Phys-Condens. Mat. 2009, 21,395502 .

45. Deslippe, J.; Samsonidze, G.; Strubbe, D. A.; Jain, M.; Cohen, M. L.; Louie, S. G., BerkeleyGW: A Massively Parallel Computer Package for the Calculation of the Quasiparticle and Optical Properties of Materials and Nanostructures. Comput. Phys. Commun. 2012, 183, 1269-1289.

46. Schlipf, M.; Gygi, F., Optimization Algorithm for the Generation of ONCV Pseudopotentials. Comput. Phys. Commun. 2015, 196, 36-44.

47. Ismail-Beigi, S., Truncation of Periodic Image Interactions for Confined Systems. Phys. Rev. B 2006, 73, 233103.

48. Hybertsen, M. S.; Louie, S. G., Electron Correlation in Semiconductors and Insulators: Band-Gaps and Quasi-Particle Energies. Phys. Rev. B 1986, 34, 5390-5413. 49. Deslippe, J.; Samsonidze, G.; Jain, M.; Cohen, M. L.; Louie, S. G., CoulombHole Summations and Energies for GW Calculations with Limited Number of Empty Orbitals: a Modified Static Remainder Approach. Phys. Rev. B 2013, 87, 165124.

50. Marini, A.; Hogan, C.; Grunig, M.; Varsano, D., Yambo: an Ab Initio Tool for Excited State Calculations. Comput. Phys. Commun. 2009, 180, 1392. 


\section{Supplementary Information for:}

\section{Point Defects and Localized Excitons in 2D WSe2}

Yu Jie Zheng ${ }^{1,2, \dagger}$, Yifeng Chen ${ }^{2, \dagger}$, Yu Li Huang ${ }^{1,3}$, Pranjal Kumar Gogoi ${ }^{1}$, Ming-Yang Li ${ }^{4}$,

Lain-Jong Li $i^{4}$, Paolo E. Trevisanutto ${ }^{2}$, Qixing Wang ${ }^{1}$, Stephen J. Pennycook, ${ }^{5,}$, Andrew T. S.

Wee $e^{1,2, *}$, Su Ying Quek $k^{1,2, *}$

${ }^{1}$ Department of Physics, National University of Singapore, 2 Science Drive 3, 117551,

Singapore

${ }^{2}$ Centre for Advanced 2D Materials, National University of Singapore, Block S14, Level 6, 6

Science Drive 2, 117546, Singapore

${ }^{3}$ Institute of Materials Research \& Engineering (IMRE), A*STAR (Agency for Science,

Technology and Research), 2 Fusionopolis Way, Innovis, 138634, Singapore

${ }^{4}$ Physical Sciences and Engineering, King Abdullah University of Science and Technology,

Thuwal, 23955-6900, Saudi Arabia

${ }^{5}$ Department of Materials Science \& Engineering, National University of Singapore, 9

Engineering Drive 1, Singapore 117575

t: These authors contributed equally to this work.

Corresponding Authors *: phyqsy@nus.edu.sg (theory); phyweets@nus.edu.sg (STM); msepsj@nus.edu.sg (STEM) 


\section{METHODS}

1. Density Functional Theory (DFT) calculations 3

2. Chemical Vapor Deposition (CVD) 5

3. Scanning tunneling microscopy/spectroscopy (STM/S) 5

4. Scanning Transmission Electron Microscopy (STEM) 5

\section{FIGURES}

FIG. S1. Formation energies of intrinsic defects. 7

FIG. S2. Side view of the intrinsic point defects in $\mathrm{WSe}_{2} \mathrm{ML}$ on graphite. 8

FIG. S3. PDOS on the intrinsic defect sites in $\mathrm{WSe}_{2} \mathrm{ML}$ on graphite. 9

FIG. S4. Atomic structure and DOS for the final state in Figure 2a of the main text. 10

FIG. S5. $\mathrm{O}_{2}$ dissociative adsorption at the Se vacancy site. $\quad 11$

FIG. S6. STM/S results for perfect SL-WSe2 on graphite. 12

FIG. S7. STM images for $\mathrm{D}_{2}$, compared with simulated STM images for $\mathrm{O}_{\text {ins }}$ and $\mathrm{O}_{\text {Se-down }}(\mathrm{O}$ substituting the bottom Se atom). 13

FIG. S8. Simulated STM images of the intrinsic defects in SL-WSe 2 on graphite. 14 FIG. S9. HAADF STEM image at $60 \mathrm{kV}$ accelerating voltage together with intensity analysis. $\quad 15$

FIG. S10. Charge density plots for DFT eigenfunctions at the K point, in the $4 \mathrm{x} 4$ supercell with $\mathrm{O}_{\text {ins. }} 16$

FIG S11. (a) GW and (b) DFT-SOC band structure with $\mathrm{m}_{z}$ projection for $4 \mathrm{x} 4$ supercell with Ose defect. 17

FIG S12. (a) GW and (b) DFT-SOC band structure with $\mathrm{m}_{\mathrm{z}}$ projection for $4 \mathrm{x} 4$ supercell with Sew defect. 17

FIG. S13. GW-BSE absorption spectra with spin orbit coupling applied perturbatively. 18

FIG S14. RPA spectra without SOC for $7 \times 7$ supercell of (a) Oins, (b) Ose, and (c) Sew anti-site defect. 19

FIG S15: STM images simulated with HSE06 functional. 20

FIG. S16: DFT-PBE band structure of Oins defective system in $4 x 4$ and $7 x 7$ supercells. 20

FIG. S17: SOC effects on the electronic and optical spectra of pristine $\mathrm{WSe}_{2}$

\section{TABLES:}

Table S1. Formation energies of selected defects in $\mathrm{WSe}_{2} .7$

Table S2. Formation energies in eV of selected defects in isolated $\mathrm{MoS}_{2} \mathrm{ML} .8$

Table S3. Energy barrier for dissociation of $\mathrm{O}_{2}$ on $\mathrm{Se}_{\text {vac }}$ in $\mathrm{WSe}_{2}$, computed for different exchange-correlation functionals. $\quad 10$

Table S4. Binding energies $\left(E_{b}\right)$ of the $\mathrm{O}$ atoms in the final state. 11

Table S5. Analysis of exciton wavefunctions. 16 


\section{METHODS}

\section{Density Functional Theory (DFT) calculations}

\section{Electronic Properties of Defects}

Except for the calculations for optical properties, the density functional theory (DFT) calculations in this manuscript were performed with the VASP $\operatorname{code}^{1}$ using the PAW approach and a kinetic energy cutoff of $400 \mathrm{eV}$. We use the PBE exchange-correlation functional ${ }^{2}$ with Grimme's D2 $2^{3}$ correction for van der Waals (vdW) interactions for all calculations except the density of states (DOS) plots which were performed with the hybrid HSE06 ${ }^{4}$ exchange-correlation functional. Spin polarization is included for the defect structures and for oxygen. Geometry optimization is performed with a force convergence criteria of 0.01 $\mathrm{eV} / \AA$ for single layer (SL)-WSe2, and $0.05 \mathrm{eV} / \AA ̊$ for $\mathrm{SL}-\mathrm{WSe} 2$ supported on graphite. The lattice constant is fixed to that optimized for the pristine $\mathrm{WSe}_{2}$ layer, which compares well to experimental values. The total energy of bulk $\mathrm{WSe}_{2}$ is converged for the chosen energy cutoff, as well as with a Monkhorst Pack k-grid sampling of $10 \times 10 \times 4$ in the bulk WSe 2 unit cell. $\mathrm{SL}-\mathrm{WSe}_{2}$ on graphite was modeled using 3 layers of graphite, with a $3 \times 3 \mathrm{WSe}_{2}$ supercell on top of a 4 x 4 supercell of graphite and $13 \AA$ of vacuum. In this supercell, the strain on WSe 2 was $0.61 \%$ and the strain on graphite was $-0.65 \%$. Defects in SL-WSe 2 on graphite were studied using a $2 \times \sqrt{3} R 30^{\circ}$ supercell of the $\mathrm{WSe} /$ graphite supercell described above. In these defect supercells, we used a k-mesh of $2 \times 2 \times 1$ for geometry optimization and $4 \times 4 \times 1$ for DOS calculations. For defects in isolated $\mathrm{WSe}_{2}$, we used a $5 \times 5$ supercell, with a $2 \times 2 \mathrm{k}$-mesh for geometry optimization and a $6 \times 6 \mathrm{k}$-mesh for DOS calculations.

The formation energy of the (charge neutral) defects is defined as

$$
E_{f}(x)=E(x)-E_{\text {pristine }}-\sum_{i} n_{i} \mu_{i}
$$

where $E(x, q)$ and $E_{\text {pristine }}$ are the total energies of the $\mathrm{WSe}_{2} /$ graphite supercell with and without the defect, respectively. $n_{i}$ denotes the number of atoms of element $i$ that have been added $\left(n_{i}>0\right)$ or removed $\left(n_{i}<0\right)$, and $\mu_{i}$ is the chemical potential of element $i$. The chemical potentials of $\mathrm{W}$ and Se are linked by the stability of $\mathrm{WSe}_{2}$, i.e. 


$$
\mu_{W S e_{2}}=\mu_{W}+2 \mu_{S e}
$$

$\mu_{W S e_{2}}$ is the total energy per formula unit of bulk WSe 2 (the conclusions are unchanged if we use instead the total energy per formula unit of monolayer $\left.\mathrm{WSe}_{2}\right) . \mu_{W}^{\max }$ and $\mu_{S e}^{\max }$ are total energies per atom of bcc $\mathrm{W}$ metal and the molecular crystal ( $R \overline{3}$ phase) of $\mathrm{Se}_{6}$ molecules, respectively. The minimum of the Se chemical potentials can be derived from formula (2), i.e., $\mu_{S e}^{\min }=\left(\mu_{W S e_{2}}-\mu_{W}^{\max }\right) / 2$.

For formation energies of oxygen defects in the presence of oxide precursors, we have referred the chemical potential of $\mathrm{O}$ to its reservoir $\mathrm{WO}_{3}(\mathrm{P} 6 \mathrm{mmm})$.

$$
\mu_{W O_{3}}=\mu_{W}+3 \mu_{O}
$$

Similar formulas are used for the formation energies of the $\mathrm{S}_{\mathrm{vac}}$, Os and $\mathrm{O}_{\text {ins }}$ defects in $\mathrm{MoS}_{2}$. The chemical potential of Mo-rich and S-rich referred to Molybdenum (bcc) and Sulphur (alpha phase) crystals. The $\mathrm{O}$ chemical potential is taken to be that from $\mathrm{MoO}_{3}(\mathrm{Pbnm})$.

For the computation of dissociation barriers and rate constants, $5 \times 5$ supercells with $>15 \AA$ vacuum were used, with a Monkhorst-Pack k-mesh of $2 \times 2 \times 1$ and a force convergence criterion of $0.01 \mathrm{eV} / \AA$. The dissociation barrier was computed using the climbing image nudged elastic band (cl-NEB) method ${ }^{5}$. The prefactor of the reaction was estimated within harmonic transition state theory (HTST) ${ }^{6-7}$, and is given by $A=\frac{\prod_{i}^{3 N} v_{i}^{I S}}{\prod_{i}^{3 N-1} v_{i}^{T S}}$, where $\mathrm{N}$ is the number of free atoms, $v_{i}^{I S}$ and $v_{i}^{T S}$ are stable (real valued) normal mode frequencies at the initial states (IS) and transition states (TS) or saddle point. At the transition state, exactly one of the vibrational modes has an imaginary frequency. From the prefactor $A$, the rate constant $k$ is obtained by $k=A \exp \left[-\frac{E^{T S}-E^{I S}}{k_{B} T}\right] . E^{I S}$ and $E^{T S}$ are the energies at the initial and transition states. $k_{B}$ and $T$ are the Boltzmann's constant and temperature, respectively.

Besides using the PBE-D2 functional as described in the main text, we have also computed the $\mathrm{O}_{2}$ dissociation barriers using the RPBE functional ${ }^{8}$ with and without Grimme's D3 dispersion correction, ${ }^{9}$ and the BEEF-vdW functional. ${ }^{10}$ The RPBE functional, when applied to dissociative adsorption of $\mathrm{O}_{2}$ on $\mathrm{Cu}$ surfaces, gave 
reasonable agreement with experiment, ${ }^{11}$ while the BEEF-vdW functional performed well compared to an experimental benchmark database of molecular dissociation barriers on surfaces. ${ }^{12}$ The results are shown in Table S1.

DFT band structures with spin orbit coupling (SOC) were also computed using VASP.

\section{Random Phase Approximation (RPA) calculations}

RPA calculations were performed with Yambo $^{13}$ using a damping parameter of 0.01 eV. RPA with SOC calculations were performed with spinor wavefunctions using fully relativistic ONCV pseudopotentials. For $4 \times 4$ supercells, the response function has a dimension in reciprocal space of $1000 \mathrm{mHa}$. However, for $7 \times 7$ supercells, the corresponding dimension is $\sim 0.5 \mathrm{mHa}$ due to the large computational expense.

\section{Chemical Vapor Deposition (CVD)}

Monolayer $\mathrm{WSe}_{2}$ was grown directly on graphite and on sapphire substrates by chemical vapor deposition (CVD) as reported in Ref. 14. In brief, high purity $\mathrm{WO}_{3}$ and Se powders are used as precursors. $\mathrm{WO}_{3}$ powders were placed in a ceramic boat at the center of a furnace and Se powders were placed at the upstream side, while the graphite or sapphire substrate was positioned in the downstream side next to the $\mathrm{WO}_{3}$ powders. Ar was used as carrier gas to carry the evaporated $\mathrm{Se}$ and $\mathrm{WO}_{3}$ to the target substrates for reaction. $\mathrm{H}_{2}$ was added as a reducing agent. STM and STEM confirmed that the samples used were monolayer in thickness.

\section{Scanning tunneling microscopy/spectroscopy (STM/S)}

STM/S measurements were performed in a custom-built multi-chamber system housing an Omicron LT-STM operating at $\sim 77 \mathrm{~K}$ under ultrahigh vacuum conditions $\left(10^{-10} \mathrm{mbar}\right)$. All STM images were recorded in constant current mode with tunneling current in the range 50$100 \mathrm{pA}$. Differential conductance dI/dV or STS were acquired by a lock-in amplifier with a sinusoidal modulation of $40 \mathrm{mV}$ at $625 \mathrm{~Hz}$. Note that the bias voltage ( $\mathrm{V}_{\text {Tip }}$ ) is applied on the STM tip with respect to the sample, hence negative values correspond to empty states and positive values correspond to filled states. Each STS curve was obtained by averaging hundreds 
of individual spectra acquired. An electrochemically etched tungsten tip was used in all measurements. Before STM investigations, the ex-situ grown sample was degassed at $\sim 300{ }^{\circ} \mathrm{C}$ overnight to remove adsorbates (e.g., $\mathrm{O}_{2}, \mathrm{H}_{2} \mathrm{O}$, etc.) physisorbed during exposure in ambient conditions.

\section{Scanning Transmission Electron Microscopy (STEM)}

\section{Transfer to TEM grid:}

At first, the $\mathrm{WSe}_{2}$ /sapphire sample surface was spin-coated with poly(methyl methacrylate) (PMMA, A4, $950 \mathrm{~K}$ in anisole, MicroChem) at $4000 \mathrm{rpm}$ for 60 second. After curing the spincoated sample at $100^{\circ} \mathrm{C}$ for $10 \mathrm{~min}$, the edges of the sample were scratched, so that the etching agent can easily reach the WSe-sapphire interface. The sample was then floated on $\mathrm{NaOH}$ $(3 \mathrm{M})$ etching solution to separate the PMMA coated $\mathrm{WSe}_{2}$ film from the sapphire substrate. After separation, the floating film was transferred to DI water beakers a few times consecutively and later fetched using a quantifoil copper TEM grid. The TEM grid with PMMA-coated $\mathrm{WSe}_{2}$ film was thereafter heated at $100^{\circ} \mathrm{C}$ for $5 \mathrm{~min}$ to get better adhesion and to remove water. The PMMA coating was washed off finally, using acetone and IPA. This transfer process was carried out inside a class 1000 cleanroom in Singapore Synchrotron Light Source (SSLS) situated at the National University of Singapore (NUS) campus.

\section{Scanning Transmission Electron Microscopy:}

Aberration-corrected scanning transmission electron microscopy (STEM) images were taken at $60 \mathrm{kV}$ accelerating voltage using the JEOL ARM200 F installed inside SSLS, NUS. The microscope, with $80 \mathrm{pm}$ resolution at $200 \mathrm{kV}$ and demonstrated information transfer of $95 \mathrm{pm}$ at $40 \mathrm{kV}$, is capable of high-resolution imaging to reveal the atomic structure and defects. The lower accelerating voltage of $60 \mathrm{kV}$ used here for $\mathrm{WSe}_{2}$ is much below the knock-on damage threshold of Se and $\mathrm{W}$ atoms in $\mathrm{WSe}_{2}$, and hence it ensures negligible beam-damage. Highangle annular dark-field (HAADF) image intensities depend on the atomic number of the corresponding atoms in the sample. All the images reported here were taken with the HAADF detector with the collection angle range of $68-280 \mathrm{mrad}$. The convergence angle of the probe beam was about $30 \mathrm{mrad}$. 

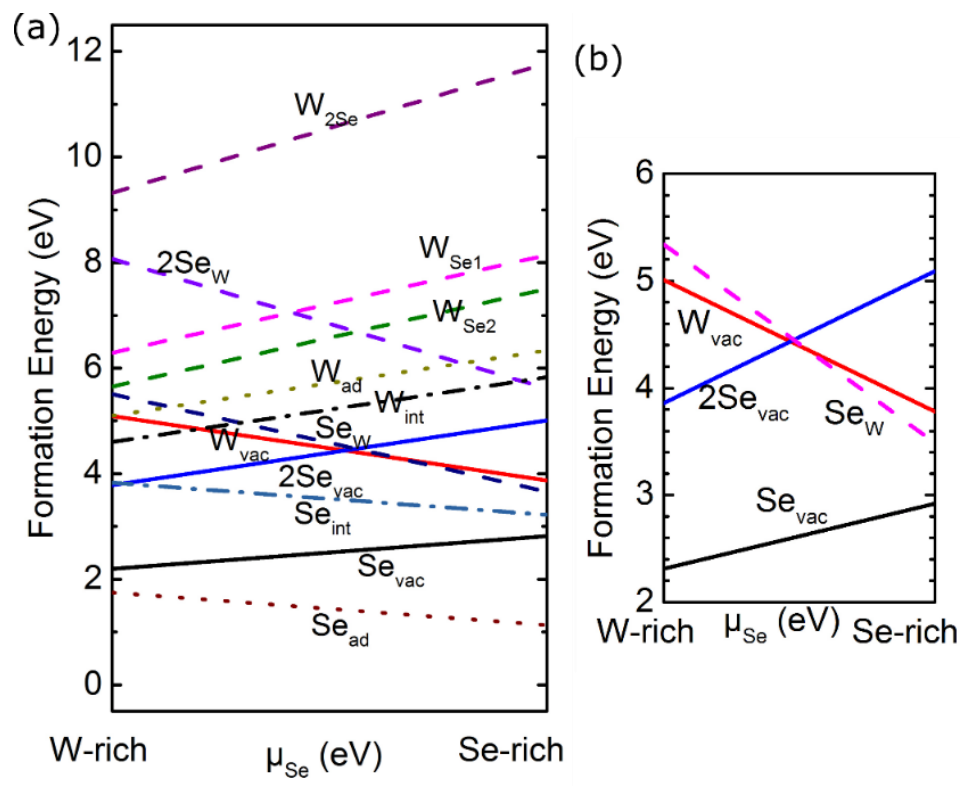

FIG S1. Formation energies of intrinsic defects (a) with and (b) without graphite substrate.

Solid lines denote vacancies, dashed lines substitutional (antisite) defects, dotted lines adatoms, and dotted-dashed lines intercalated atoms.

\begin{tabular}{|l|c|c|c|c|}
\hline \multirow{2}{*}{ Defect } & \multicolumn{2}{|c|}{ W-rich conditions } & \multicolumn{2}{c|}{ Se-rich conditions } \\
\cline { 2 - 5 } & Isolated & Supported & Isolated & Supported \\
\hline Se $_{\text {vac }}$ & 2.31 & 2.20 & 2.92 & 2.82 \\
\hline $\mathrm{W}_{\text {vac }}$ & 5.01 & 5.09 & 3.78 & 3.87 \\
\hline Sew & 5.34 & 5.51 & 3.49 & 3.66 \\
\hline $2 S_{\text {vac }}$ & 3.86 & 3.78 & 5.09 & 5.01 \\
\hline OSe $_{\text {nyyyy}}$ & 0.52 & 0.33 & 0.73 & 0.53 \\
\hline$O_{\text {ins }}$ & 2.38 & 2.36 & 1.97 & 1.95 \\
\hline$O_{\text {ad }}$ & 2.91 & 2.81 & 2.50 & 2.40 \\
\hline
\end{tabular}

Table S1. Formation energies in eV of selected intrinsic defects, and O-related defects in isolated $\mathrm{WSe}_{2} \mathrm{ML}$ and in $\mathrm{WSe}_{2} \mathrm{ML}$ supported on graphite. 


\begin{tabular}{|l|c|c|}
\hline \multicolumn{1}{|c|}{ Defect } & Mo-rich conditions & S-rich conditions \\
\hline $\mathrm{S}_{\mathrm{vac}}$ & 1.55 & 2.81 \\
\hline Os & -0.09 & 0.33 \\
\hline Oins $_{\text {ins }}$ & 3.72 & 2.88 \\
\hline
\end{tabular}

Table S2. Formation energies in eV of selected defects in isolated $\mathrm{MoS}_{2} \mathrm{ML}$.

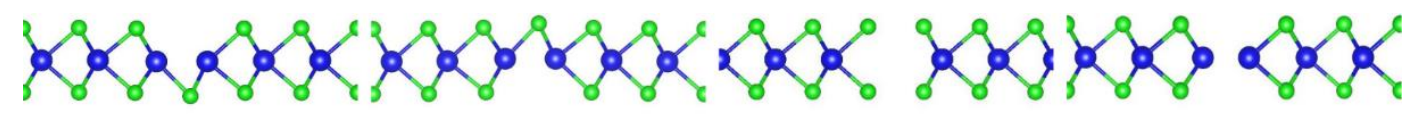
(a) Se1 $1_{\mathrm{vac}}^{\infty}$
(b) $\mathrm{Se} 2_{\mathrm{vac}}$
(c) $\mathrm{W}_{\mathrm{vac}}^{\infty}$
(d) $2 \mathrm{Se}_{\mathrm{vac}}$

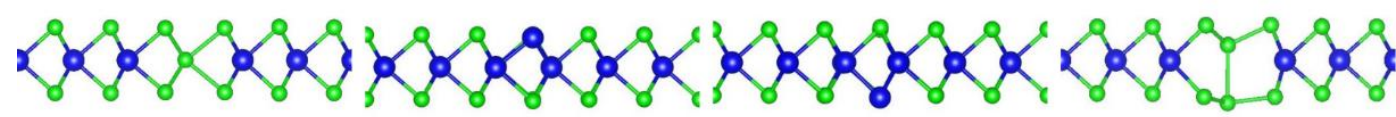

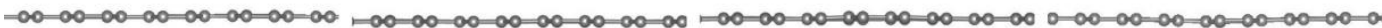
(e) $\mathrm{Se}_{\mathrm{W}}$
(f) $\mathrm{W}_{\mathrm{Se} 1}^{-\infty}$
$(\mathrm{g}) \mathrm{W}_{\mathrm{Se} 2}$
(h) $2 \mathrm{~S}_{\mathrm{W}}^{-\infty}$

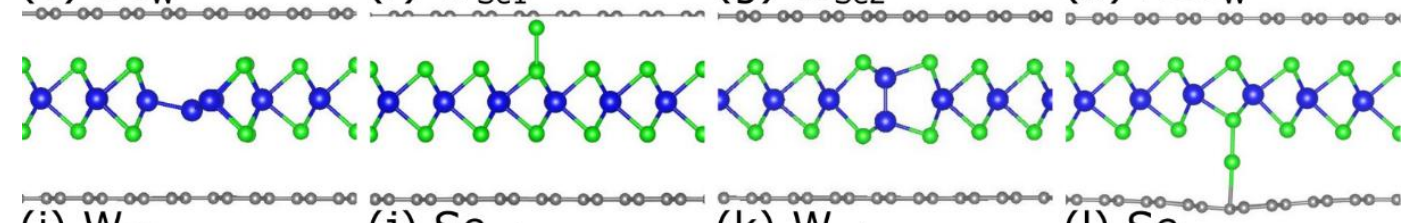

(i) $W_{25}$

(j) $\mathrm{Se}_{\text {ad }}$

(k) $W_{a d}$

(1) $\mathrm{Se}_{\text {int }}$

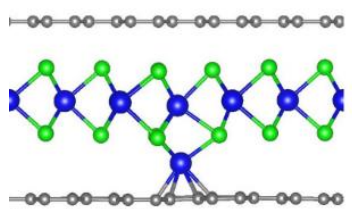

$(\mathrm{m}) \mathrm{W}_{\text {int }}$

$-\infty-\infty=\infty-\infty=\infty-\infty=\infty-\infty$

FIG. S2. Side view of the intrinsic point defects in WSe 2 ML on graphite. Blue: W, Green: Se,

Gray: C. We note that $\mathrm{W}_{\mathrm{Se} 2}$ has a slightly smaller formation energy than $\mathrm{W}_{\mathrm{Se}}$. Analysis of the detailed atomic structure indicates that the W-W bonds are $\sim 0.1 \AA$ longer in $\mathrm{W}_{\mathrm{Se} 2}$ than in $\mathrm{W}_{\mathrm{Se} 1}$, suggesting that interaction with graphite stabilizes the substitutional $\mathrm{W}$ atom. 

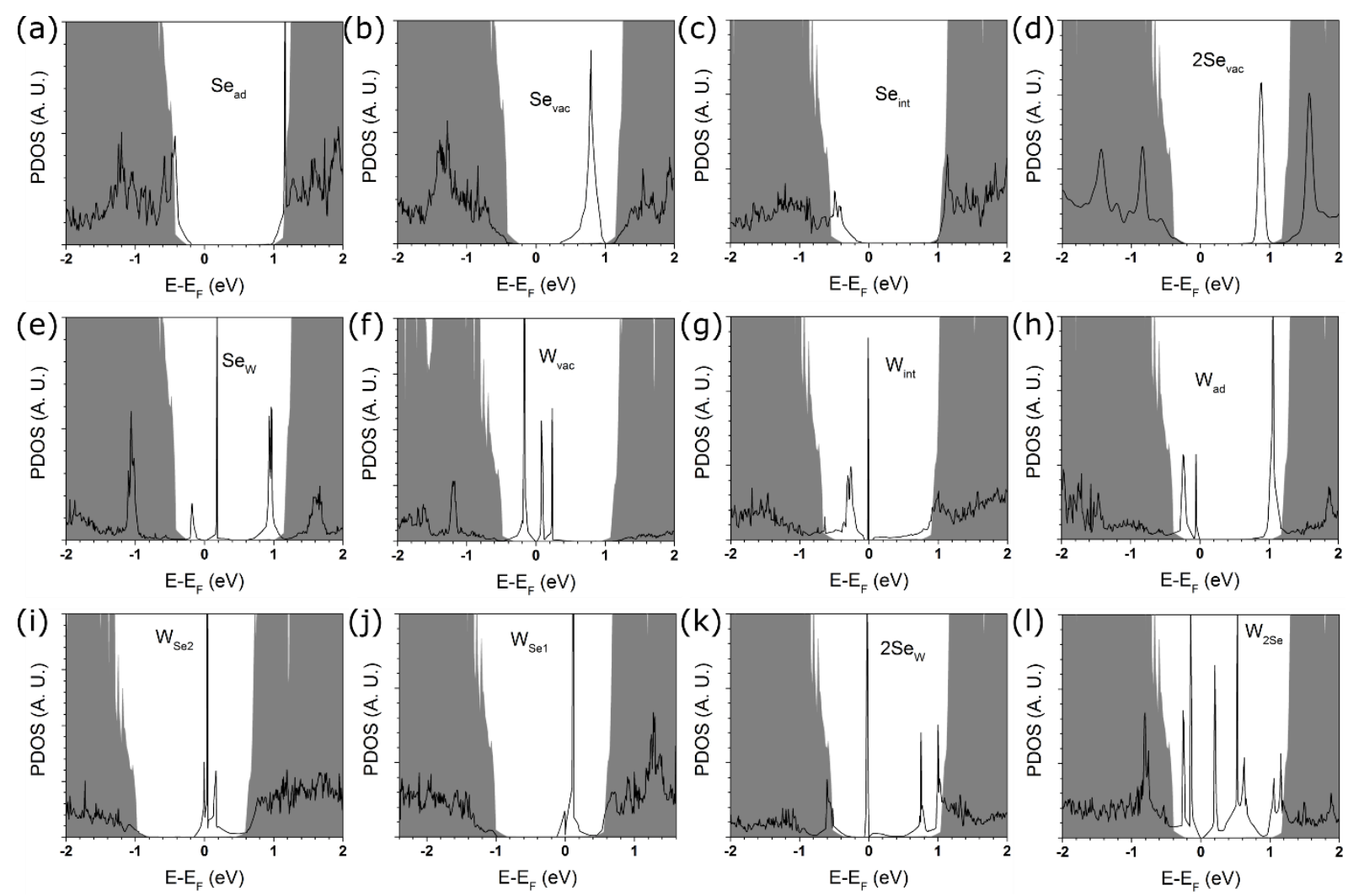

FIG. S3. PDOS (DFT PBE) on the intrinsic defect sites in $\mathrm{WSe}_{2} \mathrm{ML}$ on graphite. Gray shading: PDOS of perfect WSe2 on graphite. The PDOS with and without defects are aligned by the 1s core level of W furthest from the defect site. The Fermi level refers to the Fermi level of WSe2 ML with defects on graphite. 
Table S3. Energy barrier for dissociation of $\mathrm{O}_{2}$ on $\mathrm{Se}_{\text {vac }}$ in $\mathrm{WSe}_{2}$, computed for different exchange-correlation functionals.

\begin{tabular}{|l|c|c|c|c|c|}
\hline & PBE-D2 & $\begin{array}{c}\text { PBE-D2 } \\
\text { (with zero } \\
\text { point } \\
\text { correction) }\end{array}$ & RPBE & RPBE-D3 & BEEF-vdW 1 \\
\hline Barrier $(\mathrm{eV})$ & 0.52 & 0.48 & 0.54 & 0.57 & 0.51 \\
\hline
\end{tabular}

1. For BEEF-vdW, the number in the table is obtained from structures where the lattice constant is fixed to that of PBE-D2, which is closer to experiment. Using the lattice constant optimized with BEEF-vdW (overestimated by $\sim 3.5 \%$ ), we obtain a much smaller energy barrier of $0.13 \mathrm{eV}$.
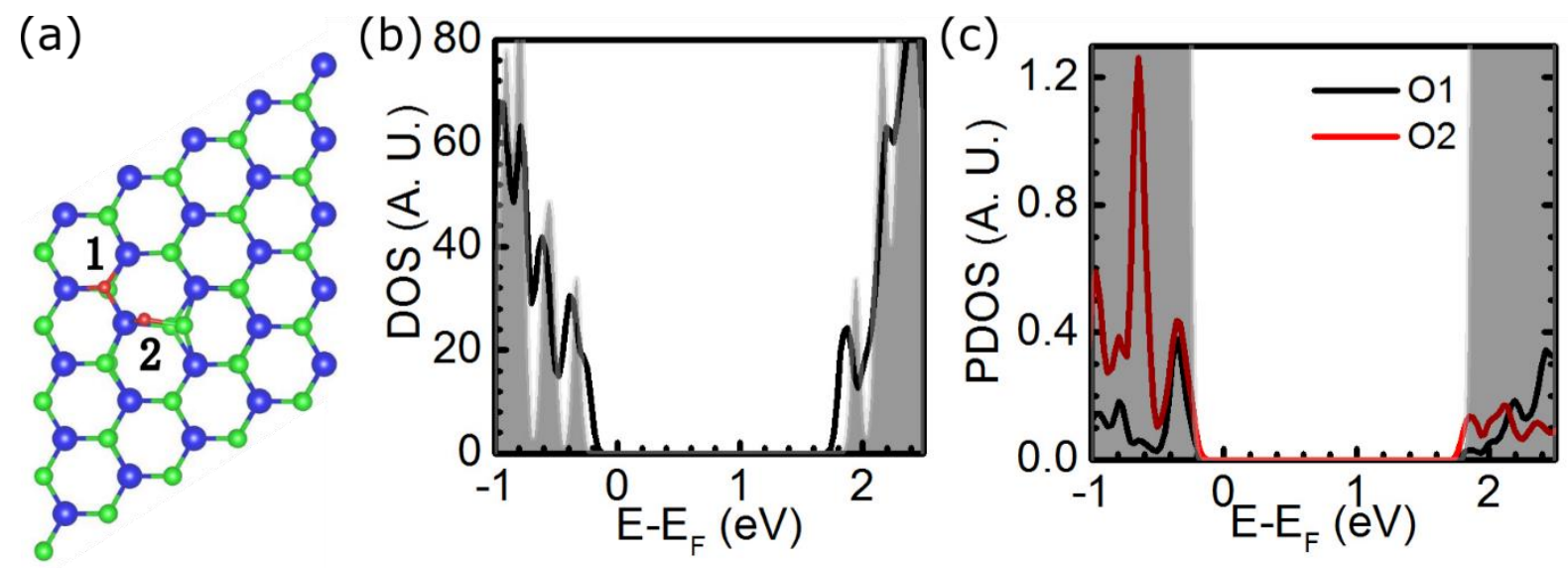

FIG. S4. Atomic structure and DOS for the final state in Figure 2a of the main text. (a) Top view of atomic structure (Blue: W, Green: Se, Red: O), (b) DOS of final state, (c) PDOS on the $\mathrm{O}$ atoms which are marked in (a). Gray shading represents the DOS of perfect WSe 2 , aligned using the 1s levels of $\mathrm{W}$ atoms furthest from the defect. 
Table S4. Binding energies $\left(E_{b}\right)$ in $\mathrm{eV}$ of the $\mathrm{O}$ atoms in the final state. $E_{b}=E_{2 O}-E_{1 O}-\mu_{O}$, where $E_{2 O}$ is the total energy of the final state in Figure $2 \mathrm{a}, E_{10}$ is the total energy after the specified $\mathrm{O}$ atom is removed, and $\mu_{O}$ is half of the total energy of $\mathrm{O}_{2}$, and for the number in brackets, $\mu_{O}$ is the energy of atomic O. Spin polarization is included. We see that the $\mathrm{O} 1$ atom is much more strongly bound that $\mathrm{O} 2$.

\begin{tabular}{|c|c|c|}
\hline & $\mathrm{O} 1$ & $\mathrm{O} 2$ \\
\hline$E_{b}$ & $-4.2(-6.8)$ & $0.3(-2.3)$ \\
\hline
\end{tabular}

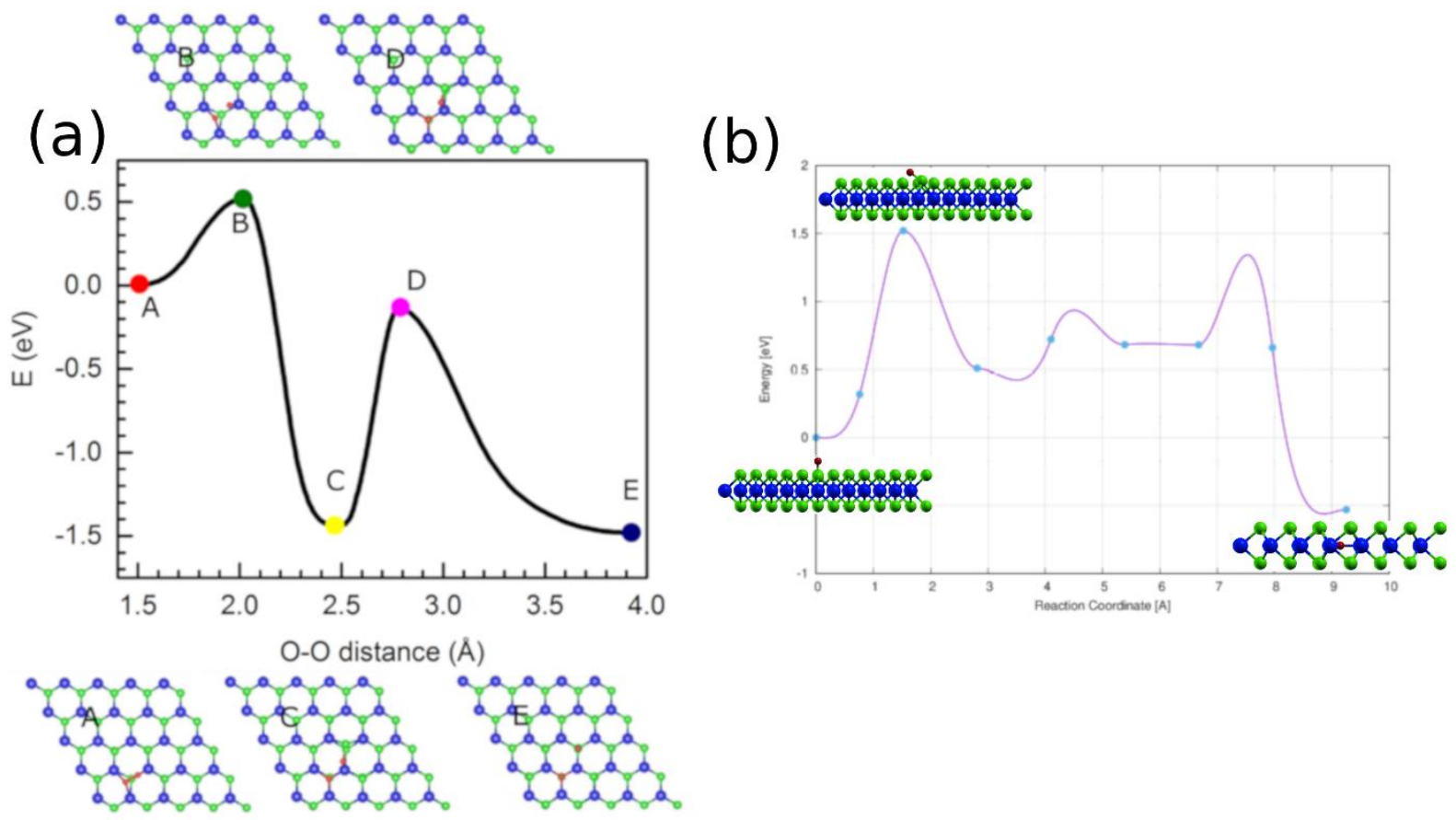

FIG. S5. (a) $\mathrm{O}_{2}$ dissociative adsorption at the Se vacancy site and diffusion of oxygen atom to (a) $\mathrm{O}_{\mathrm{ad}}$; (b) Energy barrier for $\mathrm{O}$ atom to diffuse from $\mathrm{O}_{\text {ad }}$ to $\mathrm{O}_{\text {ins. }}$. The energy barrier from $\mathrm{O}_{\mathrm{ad}}$ to $\mathrm{O}_{\text {ins }}$ is $1.5 \mathrm{eV}$, smaller than the binding energy of $\mathrm{O}$ atoms (relative to atomic $\mathrm{O}$ ) at the different binding sites. 
(a)
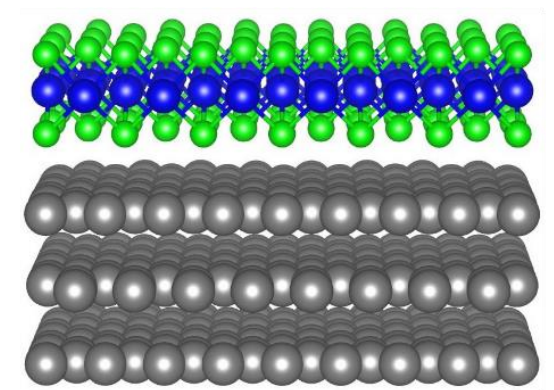

(b)

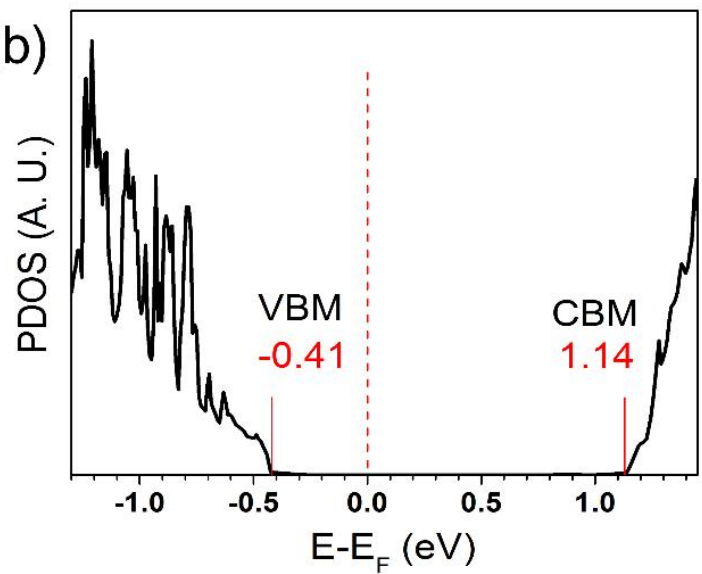

(c)

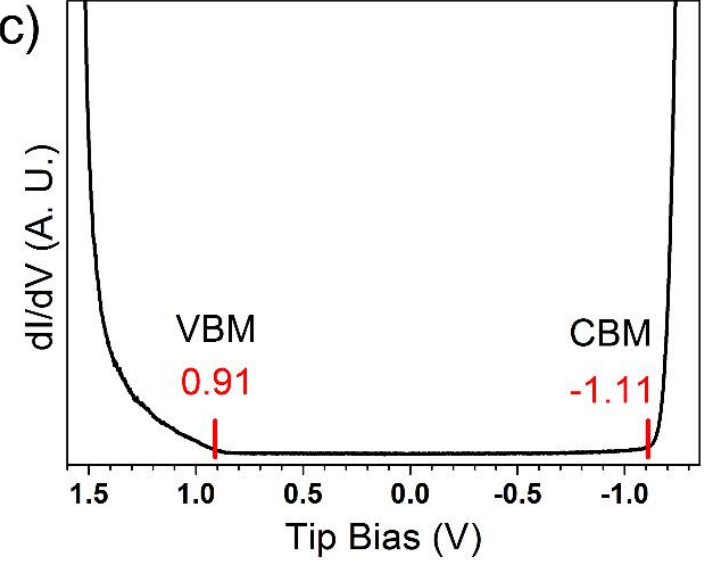

(d)
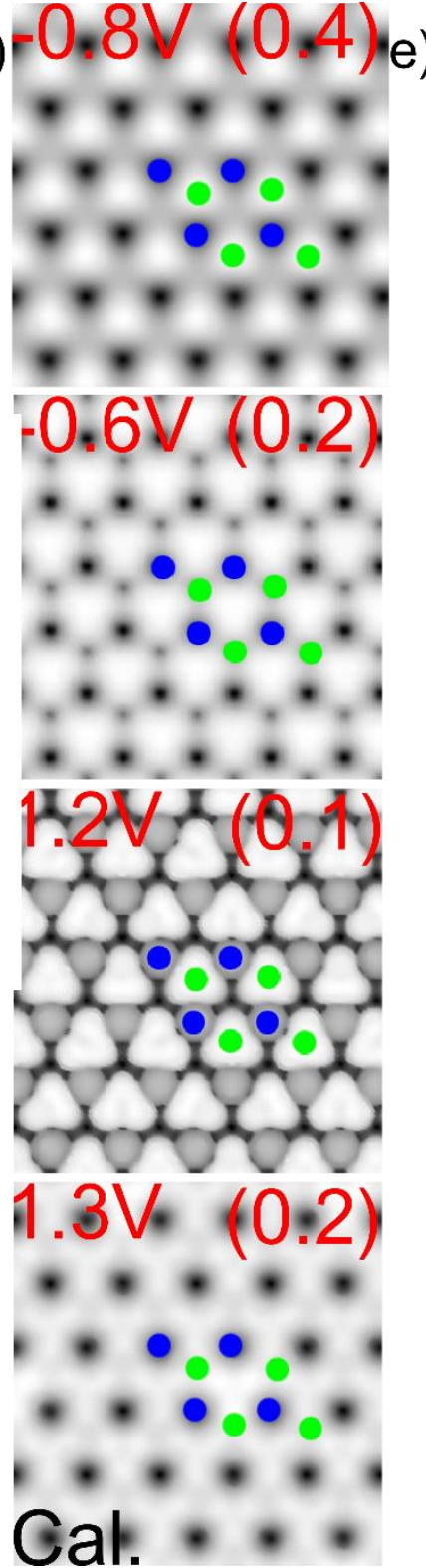
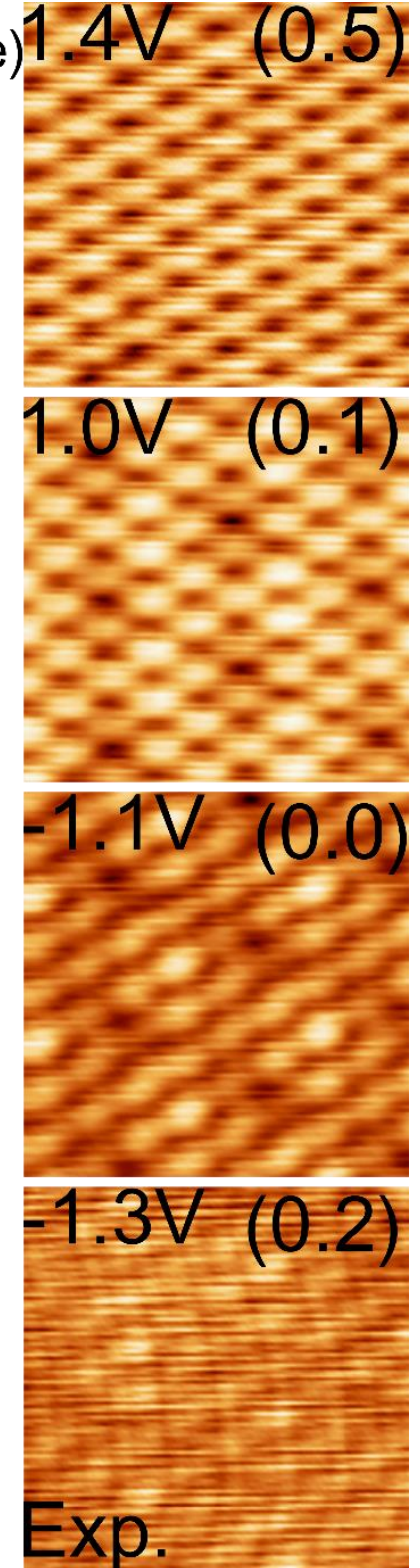

FIG. S6. STM/S results for perfect SL-WSe2 on graphite. (a) Atomic structure. (Blue: W; Green: Se; Gray: C) (b) PDOS (PBE) of $\mathrm{WSe}_{2}$ on graphite reveals a $1.55 \mathrm{eV}$ band gap. (c) STS spectrum reveals a $2.02 \mathrm{eV}$ bandgap for $\mathrm{SL}_{-} \mathrm{WSe}_{2}\left(\mathrm{~V}_{\mathrm{Tip}}=1.3 \mathrm{~V}, 68.5 \mathrm{pA}\right)$ on graphite with slight p-type doping characteristic, similar to previous reports. ${ }^{15}$ (d) Simulated STM images at different bias voltage (values in parenthesis referenced to the band edges) with atoms overlain in the images (Blue spots: W, Green spots: Se). Energy ranges chosen for the simulation approximate those in the experiment. (e) Bias dependent experimental STM images of SL$\mathrm{WSe}_{2}$ on graphite (values in parenthesis referenced to band edges). 

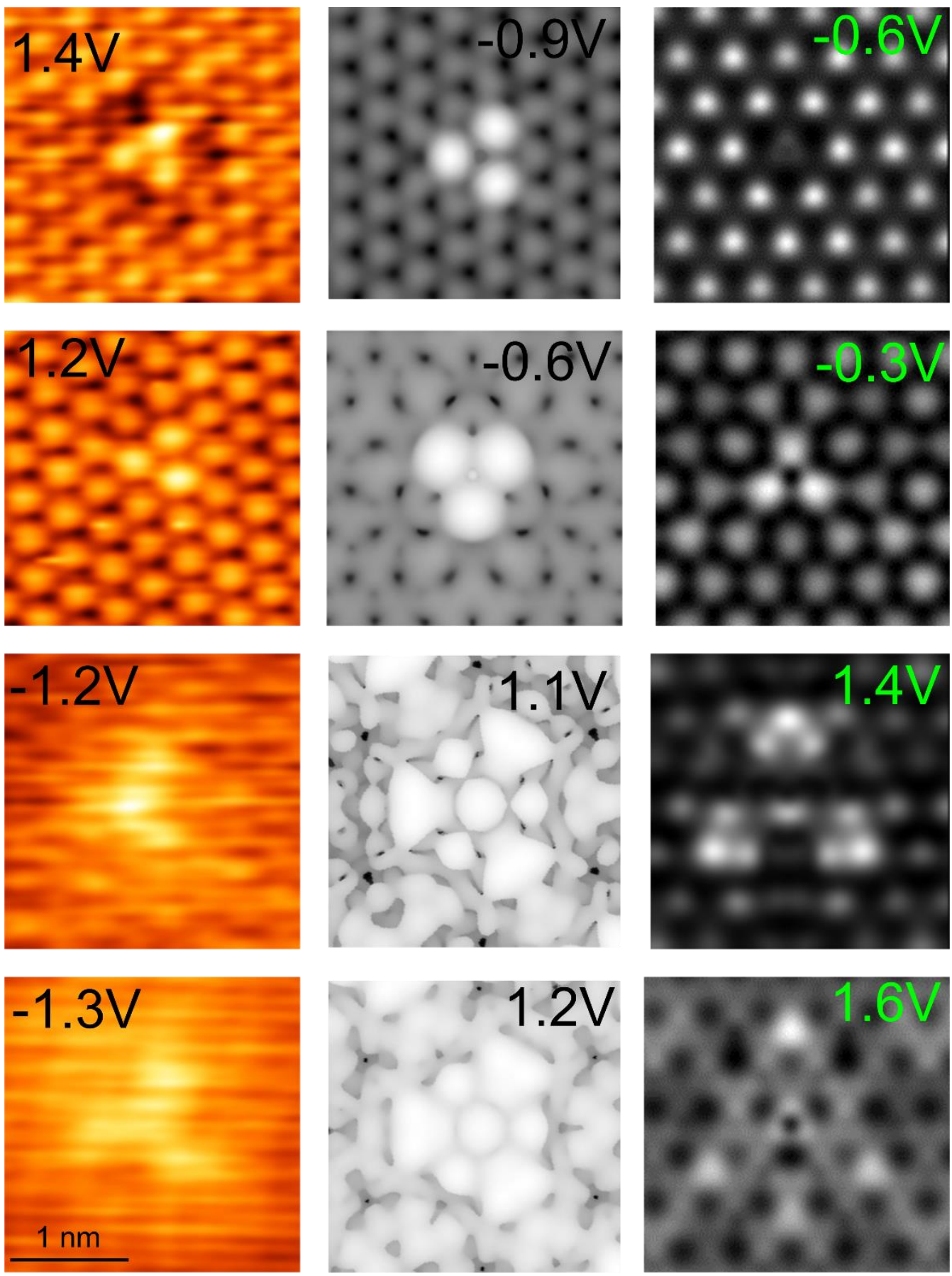

$\mathrm{D}_{2}$
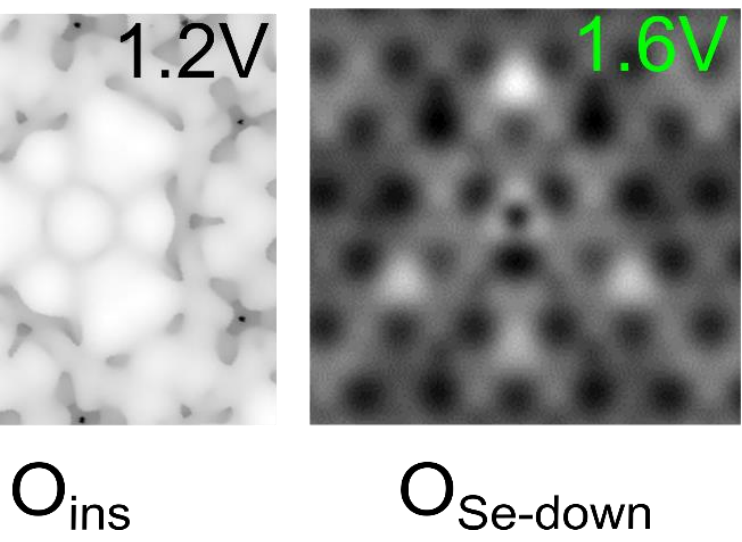

FIG. S7. STM images for $\mathrm{D}_{2}$, compared with simulated STM images for $\mathrm{O}_{\text {ins }}$ and Ose-down $(\mathrm{O}$ substituting the bottom Se atom) 
VBM-0.3 eV CBM+0.3 eV VBM-0.3 eV CBM+0.3 eV
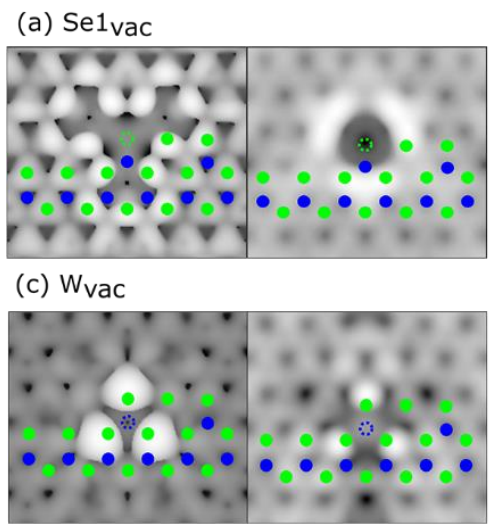

(b) $\mathrm{Se} 2 \mathrm{vac}$

(e) Sew

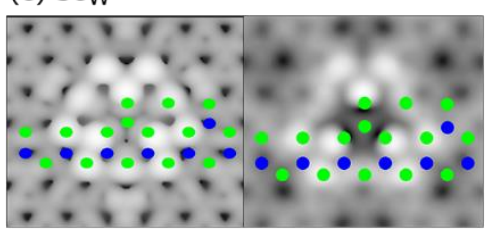

(g) WSe2

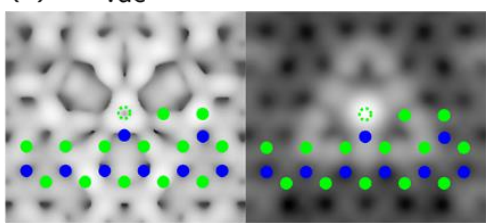

(d) $2 \mathrm{Se}_{\mathrm{vac}}$

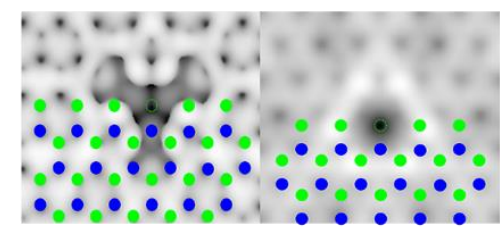

(f) WSe1

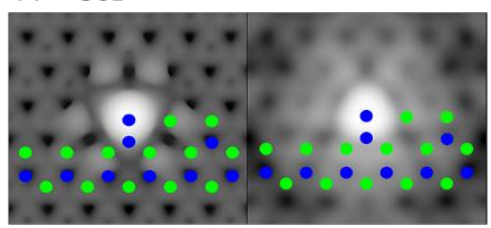

(h) 2 Sew

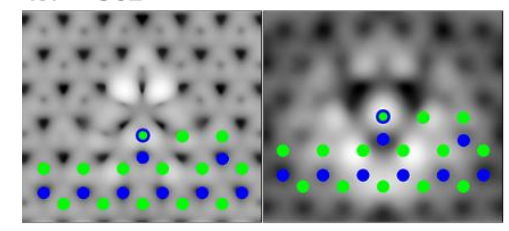

(i) $\mathrm{W}_{2} \mathrm{Se}$
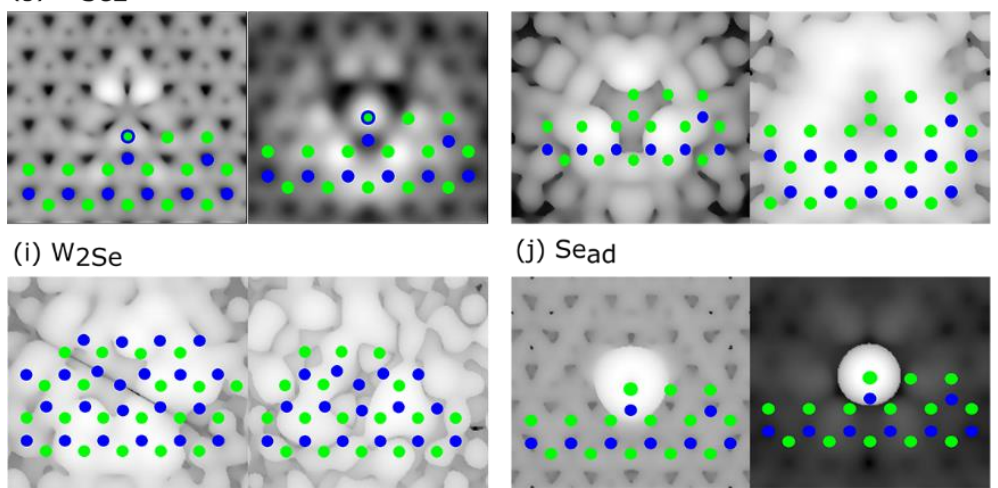

(j) Sead

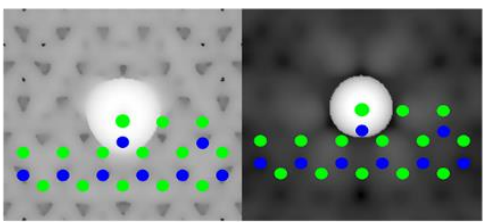

(k) Wad

(I) Seint
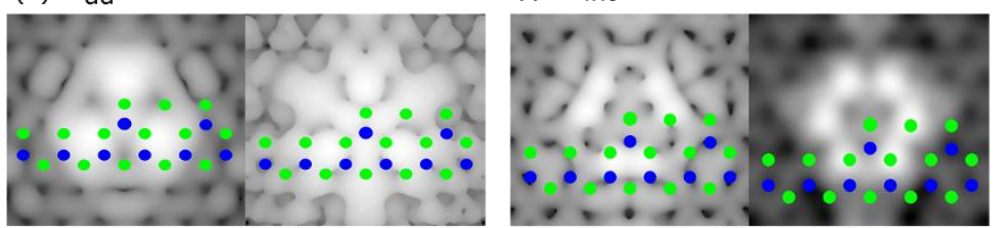

(m) Wint

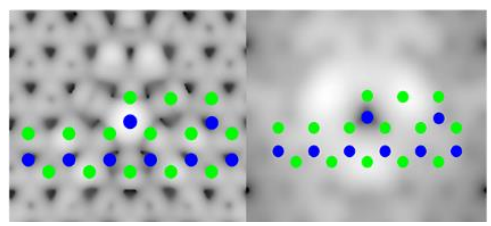

FIG. S8. Simulated STM images of the intrinsic defects in SL-WSe 2 on graphite. The energy ranges are chosen to probe occupied and unoccupied states that are $0.3 \mathrm{eV}$ away from the band edges. Atomic positions are overlain on the images. Blue: W, Green: Se. Se $1_{\text {vac }}$ and Se2 vac refer to single Se vacancies with the missing Se being in the top and bottom layer, respectively. 

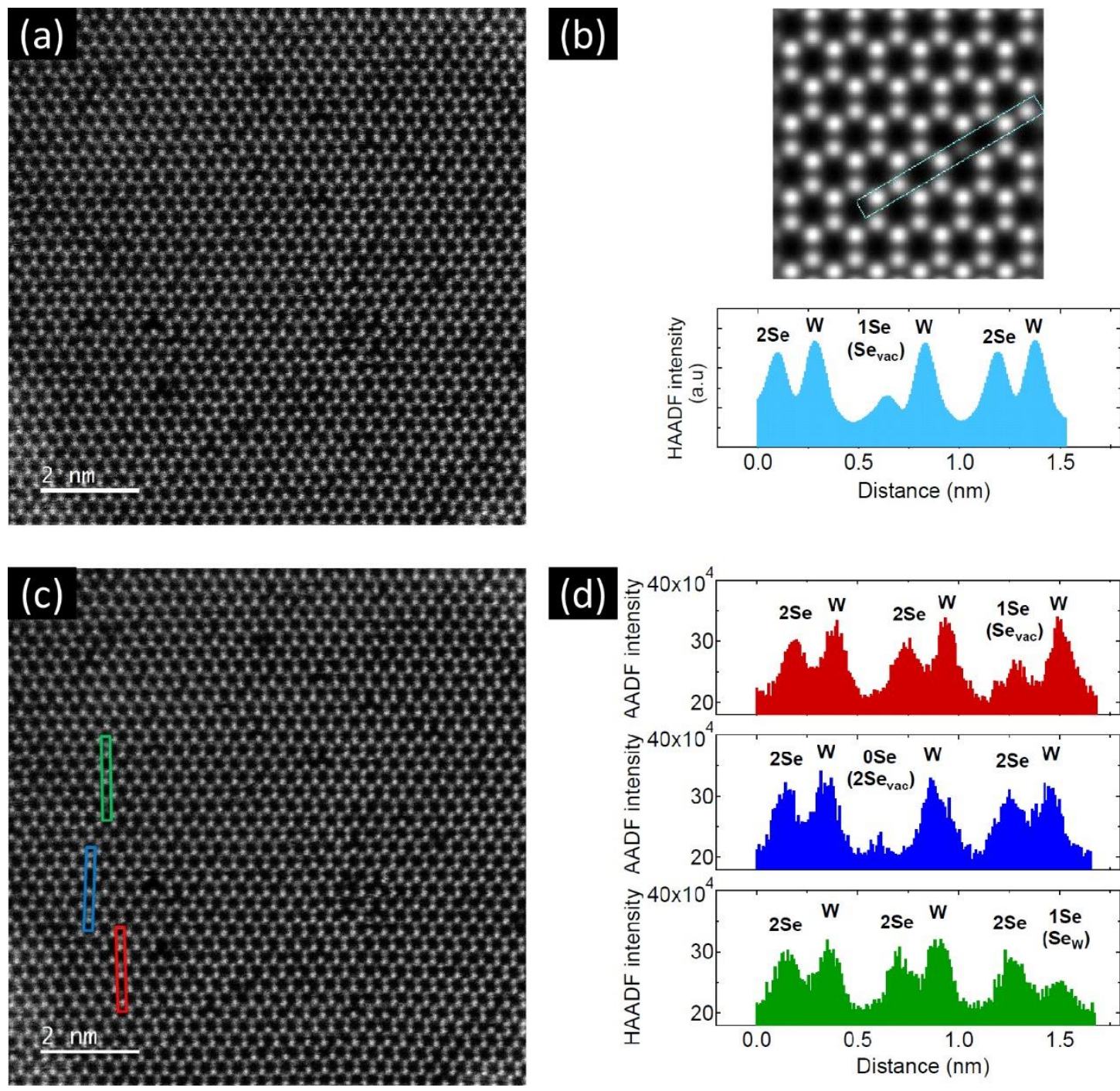

FIG. S9. HAADF STEM image at $60 \mathrm{kV}$ accelerating voltage together with intensity analysis.

(a) Raw experimental HAADF STEM image presented in the main text Fig. 4 (g) and (h) (no overlaid atoms now) is shown. (b) Simulated HAADF STEM image and intensity profile, obtained using QSTEM simulation package with input parameters same as in experiments, are shown for the representative case of a region with one $\mathrm{Se}_{\text {vac. }}$. The brighter spots are due to $\mathrm{W}$ atoms and the lesser intensity ones are due to two Se atoms at the Se site. One $\mathrm{Se}_{\mathrm{vac}}$ is in the simulated image as can be seen in the line profile. The $\mathrm{Se}_{\mathrm{vac}}$ can be easily distinguished both in the simulated image as well in the line profile due to its reduced intensity. (c) The same experimental HAADF STEM image as in (a) is shown now with line profiles indicated. The red line profile captures a $\mathrm{Se}_{\mathrm{vac}}$, the blue line profile captures a $2 \mathrm{Se}_{\mathrm{vac}}$, and the green line profile captures a $\mathrm{Se}_{\mathrm{w}}$. (d) The corresponding intensity line profiles are plotted for the regions indicated in (c). These experimental line profile intensities (taking the background also into consideration) are used to distinguish the nature of defects by comparing them with corresponding simulated image intensities quantitatively. 

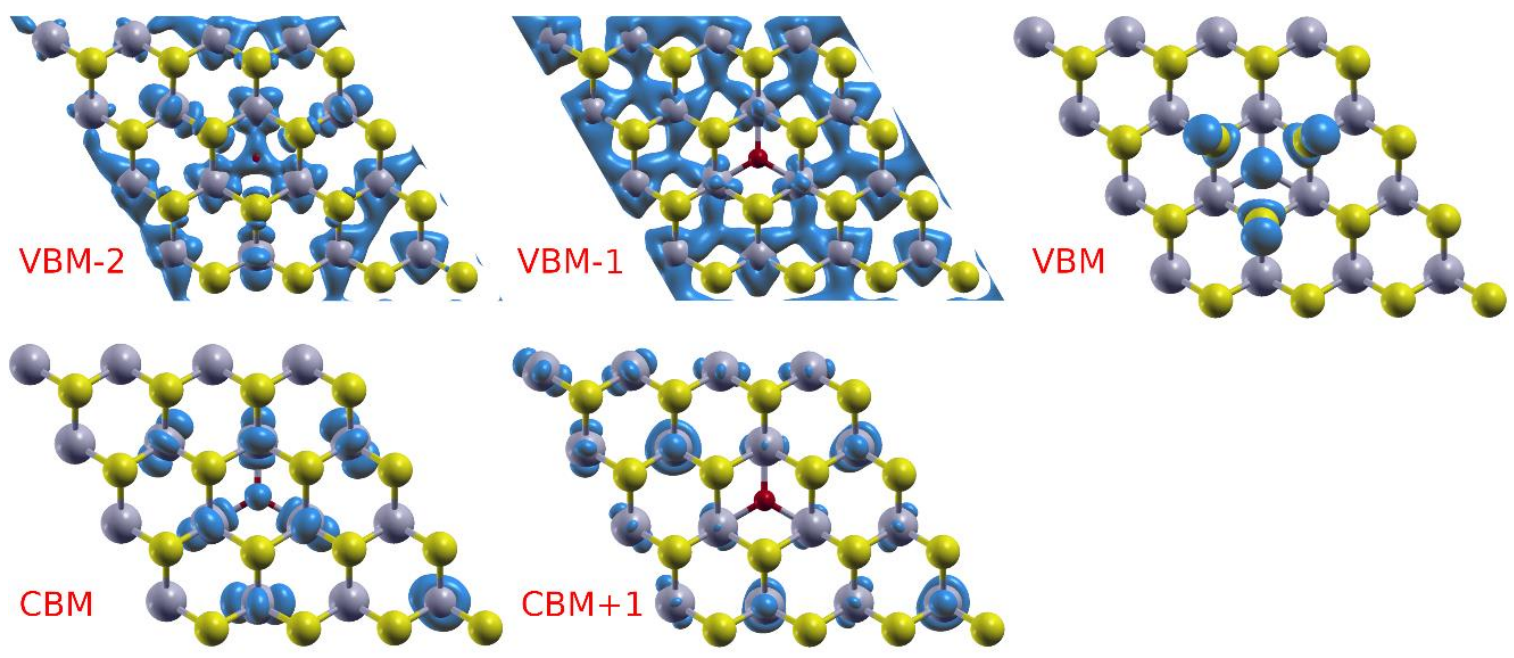

FIG. S10. Charge density plots for DFT eigenfunctions at the K point, in the $4 \times 4$ supercell

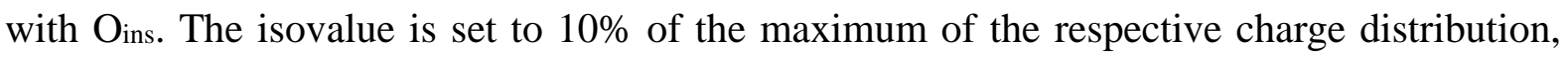
except for VBM, which is set to $1 \%$ for better visualization.

\begin{tabular}{|l|l|l|l|l|l|l|l|}
\hline $\begin{array}{l}\text { LX1: } \\
1.783 \mathrm{eV}\end{array}$ & CBM & CBM+1 & CBM+2 & $\begin{array}{l}\text { LX2: } \\
1.860 \mathrm{eV}\end{array}$ & CBM & CBM+1 & CBM+2 \\
\hline VBM & 0.00006 & 0.00000 & 0.00000 & VBM & 0.00002 & 0.00001 & 0.00000 \\
\hline VBM-1 & 0.88401 & 0.04781 & 0.00009 & VBM-1 & 0.41199 & 0.11755 & 0.00366 \\
\hline VBM-2 & 0.02064 & 0.00593 & 0.00002 & VBM-2 & 0.40391 & 0.00617 & 0.00269 \\
\hline $\begin{array}{l}\mid l \\
\text { LX3: } \\
1.880 \mathrm{eV}\end{array}$ & CBM & CBM+1 & CBM+2 & $\begin{array}{l}\text { A: } \\
1.978 ~ e V\end{array}$ & CBM & CBM+1 & CBM+2 \\
\hline VBM & 0.00065 & 0.00002 & 0.00000 & VBM & 0.00036 & 0.00015 & 0.00002 \\
\hline VBM-1 & 0.66412 & 0.08961 & 0.00271 & VBM-1 & 0.23457 & 0.42535 & 0.01096 \\
\hline VBM-2 & 0.17760 & 0.00832 & 0.00434 & VBM-2 & 0.19933 & 0.02762 & 0.01315 \\
\hline
\end{tabular}

Table S5: Analysis of exciton wavefunction for first few bright exciton states in the $4 x 4$ supercell with $\mathrm{O}_{\text {ins. }}$ The numbers shown are $\sum_{\vec{k}} A_{v c \vec{k}}^{S}$ for each combination of valence state $v$ and conduction state $c$, where the exciton wavefunction is given as $\Psi^{S}\left(\vec{r}_{e}, \vec{r}_{h}\right)=\sum_{v c \vec{k}} A_{v c \vec{k}}^{S} \psi_{c \vec{k}}\left(\vec{r}_{e}\right) \psi_{v \vec{k}}\left(\vec{r}_{h}\right)$. Major contributing entries are highlighted in red. It is important to note that the GW self-energy corrections result in a reordering of the valence states at this K point. In Table S5, all states are labeled according to the energy ordering in the meanfield DFT (PBE) calculation. At the K point, the DFT states VBM, (VBM-1) and (VBM-2) correspond, respectively, to the GW (VBM-2), GW VBM and GW (VBM-1). 

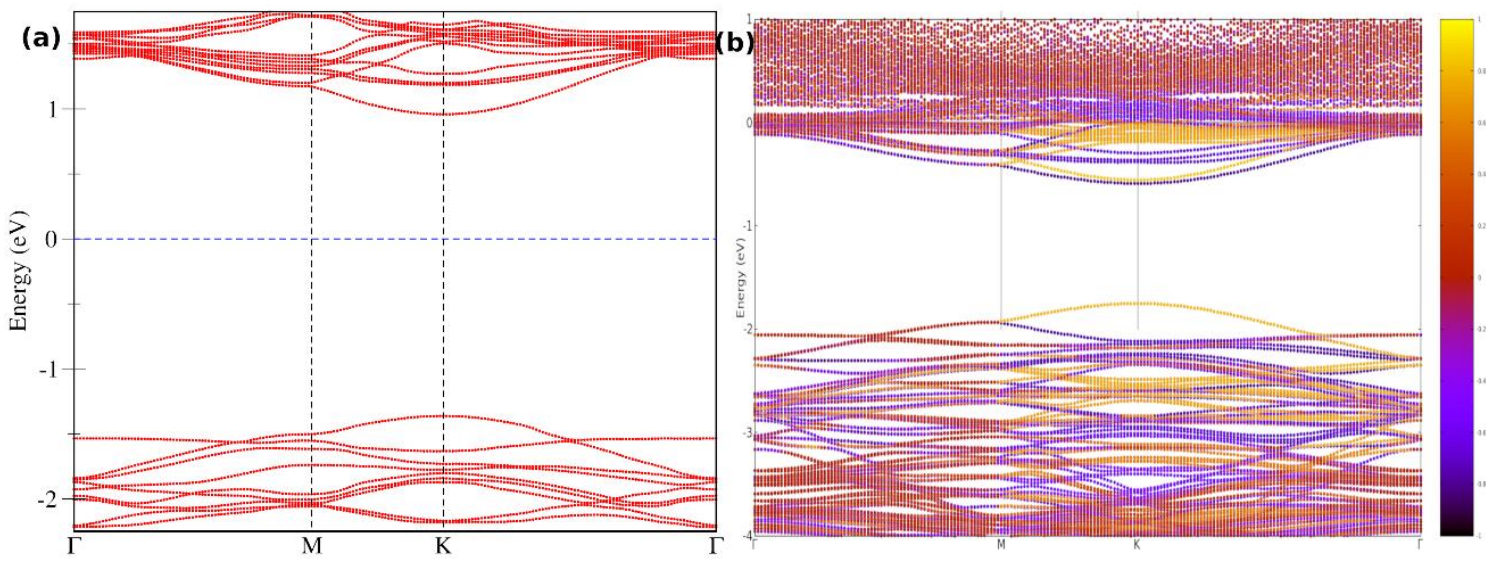

FIG S11. (a) GW and (b) DFT-SOC band structure with $\mathrm{m}_{z}$ projection for $4 \mathrm{x} 4$ supercell with Ose defect.
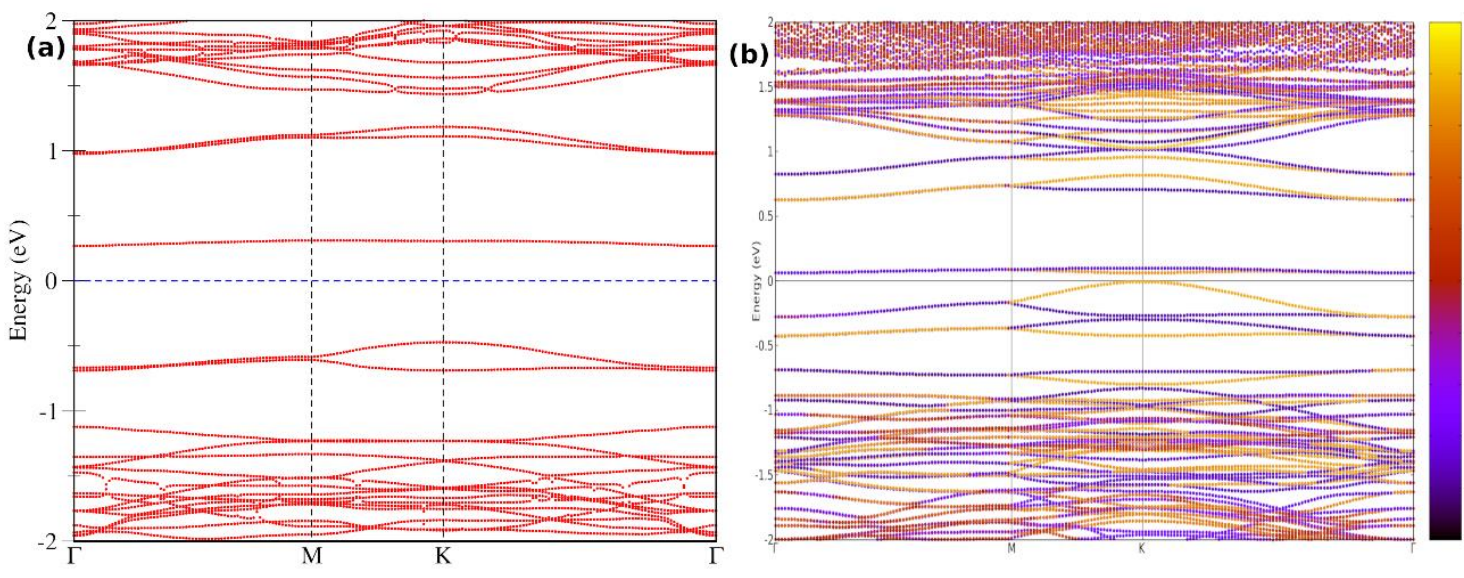

FIG S12. (a) GW and (b) DFT-SOC band structure with $\mathrm{m}_{\mathrm{z}}$ projection for $4 \mathrm{x} 4$ supercell with Sew defect. 

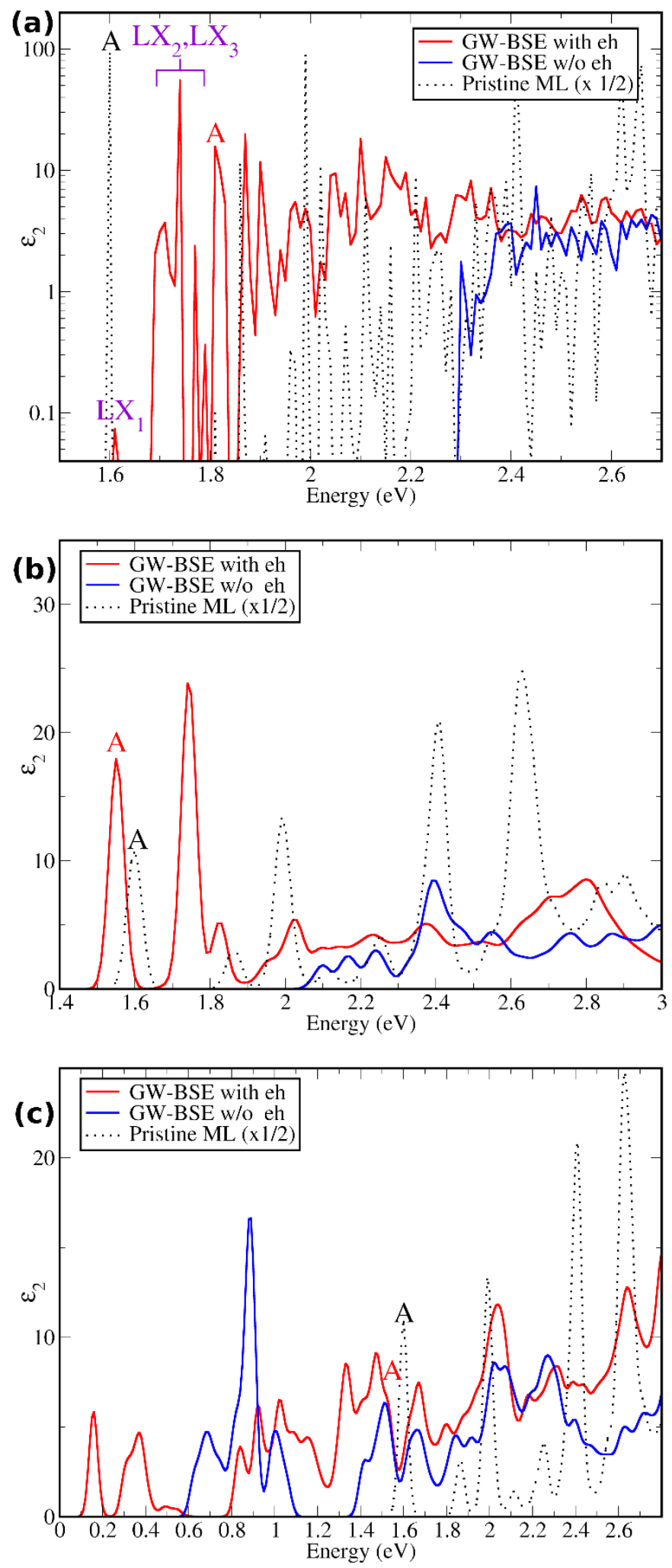

FIG. S13. GW-BSE absorption spectra with spin orbit coupling (SOC) applied perturbatively, for $4 \times 4 \mathrm{WSe}_{2}$ supercell with (a) Oins, (b) Ose and (c) Sew defect. SOC is also applied perturbatively for the independent particle absorption spectra (blue) and for the pristine $\mathrm{WSe}_{2}$ monolayer (black dashed). We note that one should focus only on peaks with energies not larger than the free exciton A peaks in each system. 

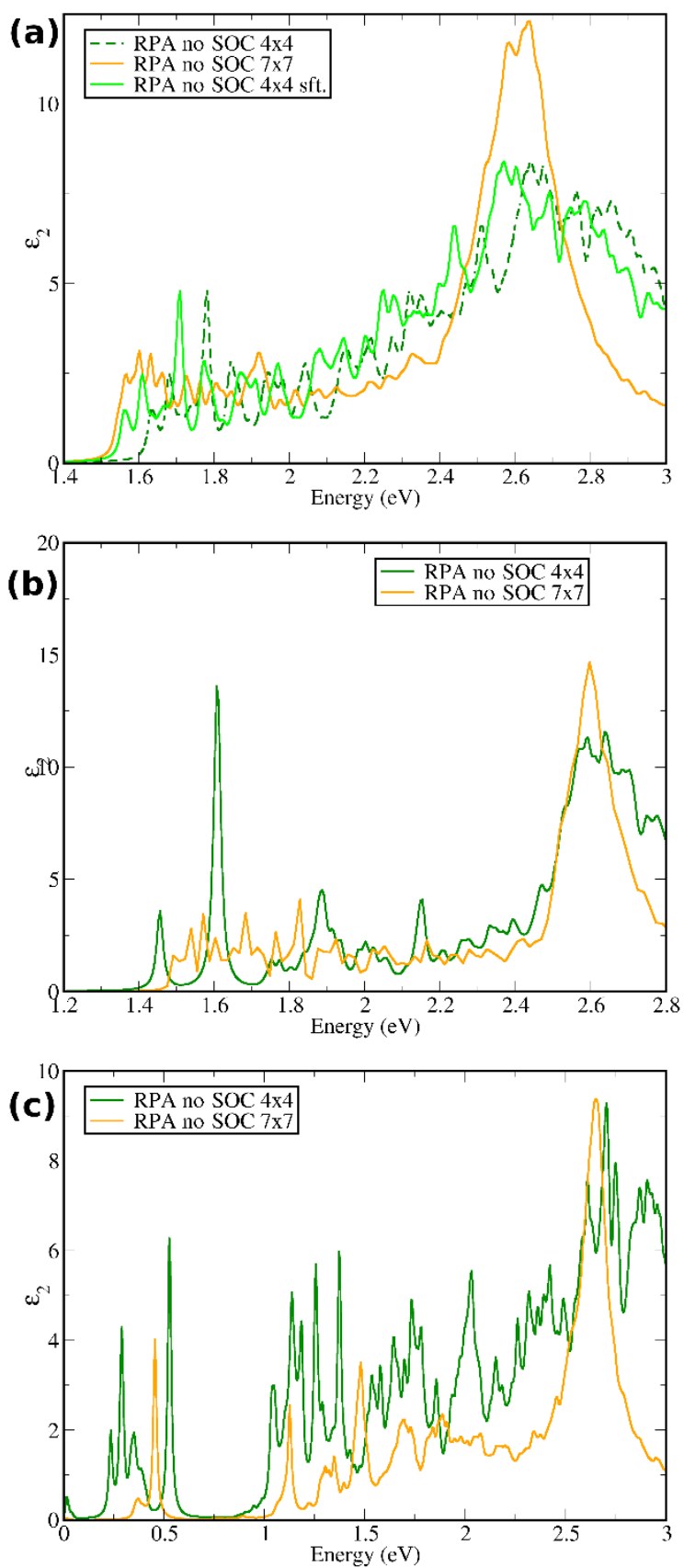

Figure S14. RPA spectra without SOC for $7 \times 7$ supercell of (a) Oins, (b) Ose, and (c) Sew antisite defect, in comparison with the corresponding $4 \times 4$ supercell result. The $0.072 \mathrm{eV}$ red shifted $4 \times 4$ spectrum in (a) is to account for the DFT gap size difference between $4 \times 4$ and $7 \times 7$ supercell systems. The fact that fewer peaks are present in the $7 \times 7$ supercells may be related to the limited number of valence and conduction bands in these calculations. 

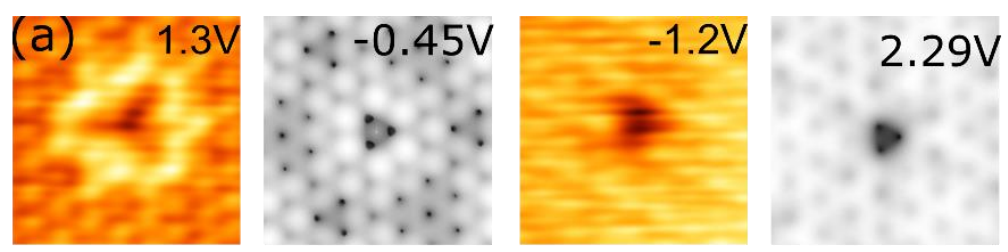

(b)
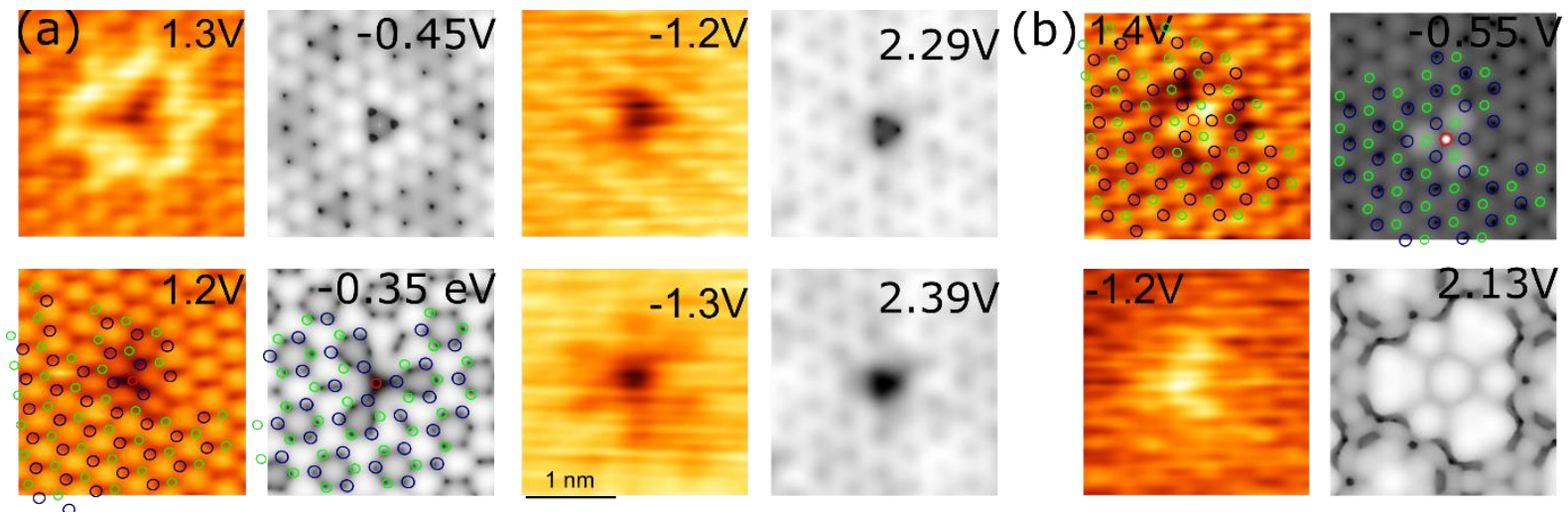

$\mathrm{D}_{1}$ vs $\mathrm{O}_{\mathrm{Se}}$

$$
D_{1} \text { vs } O_{\text {ins }}
$$

FIG. S15. STM images simulated with HSE06 functional. Left: Experimental STM (Omicron LT-STM operating at $\sim 77 \mathrm{~K}$ and $10^{-10}$ mbar) images for (a) $\mathrm{D}_{1}$ and (b) $\mathrm{D}_{2}$. Right: Simulated STM images of (a) Ose and (b) Oins using the HSE06 exchange-correlation functional. The atoms are overlain in the images for D1 and D2 defects. (Red: O; Blue: W; Green: Se).
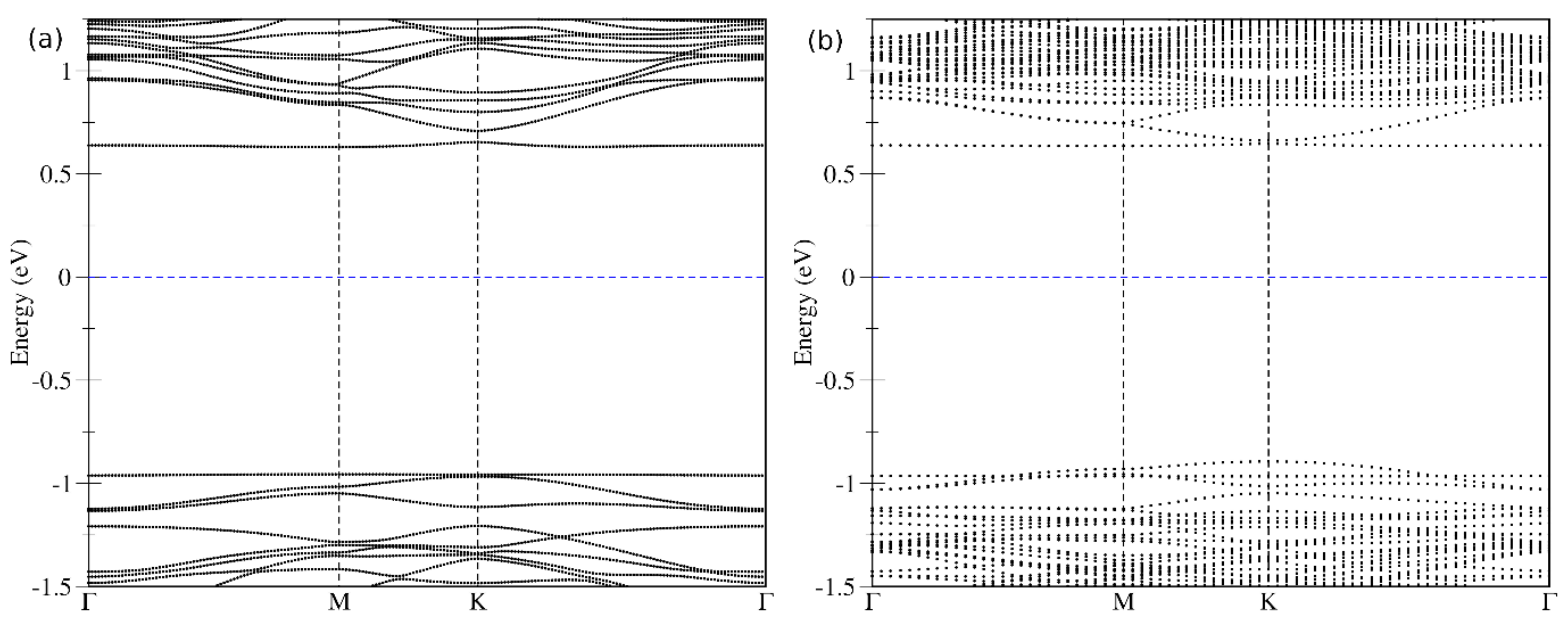

FIG. S16. DFT-PBE band structure of $\mathrm{O}_{\text {ins }}$ defective system in (a) $4 \times 4$ supercell and (b) $7 x 7$ supercell. 

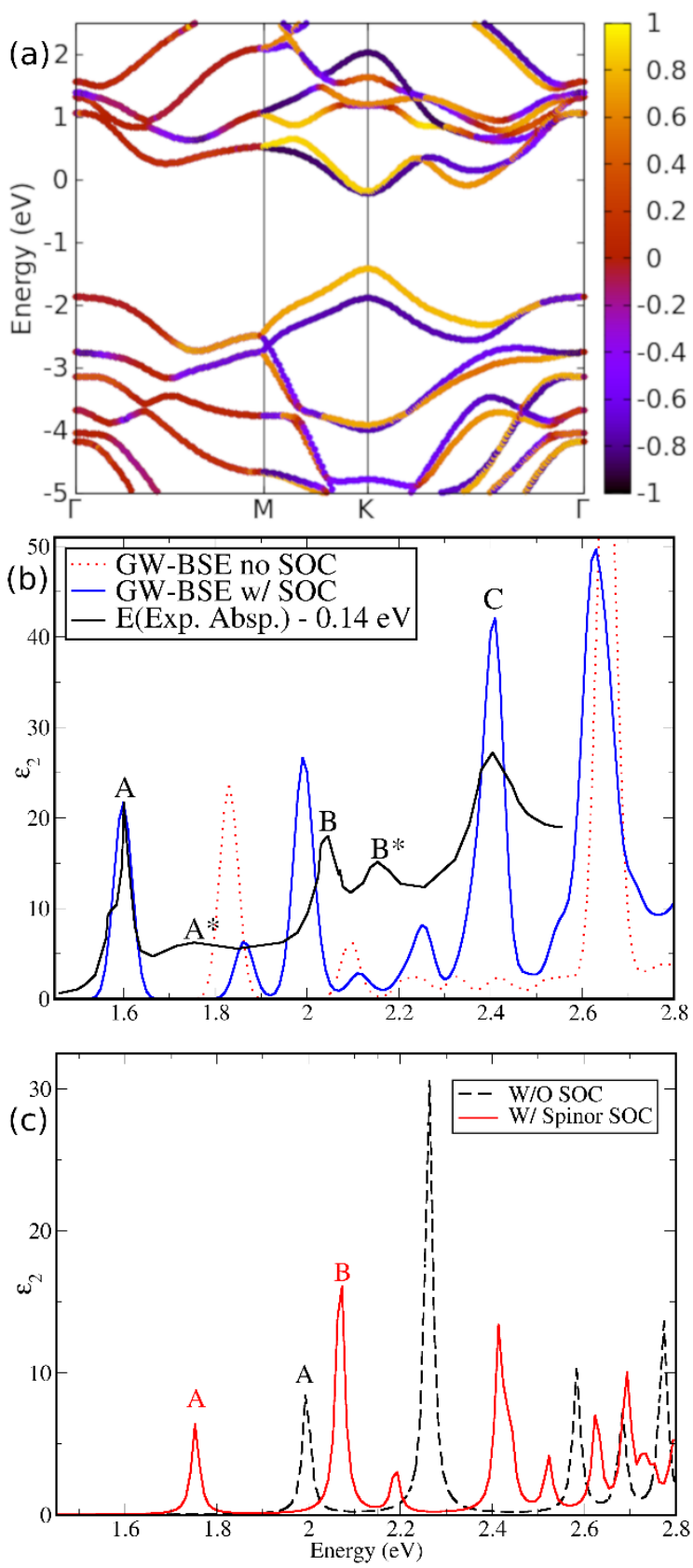

FIG. S17. SOC effects on the electronic and optical spectra of pristine $\mathrm{WSe}_{2}$ (a) PBE-SOC bandstructure of primitive cell pristine monolayer $\mathrm{WSe}_{2}$, with $\mathrm{m}_{\mathrm{z}}$ value encoded in the color depth scale. (b) GW-BSE optical absorption spectra of pristine $\mathrm{WSe}_{2}$ without and with perturbative SOC, in comparison with experimental spectrum (from BerkeleyGW). (c) GWBSE optical spectra of pristine $\mathrm{WSe}_{2}$ without and with full spinor SOC from Yambo code. Note that here the two spectra are blue shifted rigidly by the same $0.49 \mathrm{eV}$ amount to match with the experimental A peak value at $1.75 \mathrm{eV}$, due to the underlying under-converged $\mathrm{GW}$ quasiparticle spectrum for these calculations. 


\section{REFERENCES}

1. Kresse, G.; Furthmüller, J., Efficient Iterative Schemes for Ab Initio Total-Energy Calculations Using a Plane-Wave Basis Set. Phys. Rev. B 1996, 54, 11169-11186.

2. Perdew, J. P.; Burke, K.; Ernzerhof, M., Generalized Gradient Approximation Made Simple. Phys. Rev. Lett. 1996, 77, 3865-3868.

3. Grimme, S., Semiempirical GGA-Type Density Functional Constructed with a LongRange Dispersion Correction. J. Comput. Chem. 2006, 27, 1787-1799.

4. Krukau, A. V.; Vydrov, O. A.; Izmaylov, A. F.; Scuseria, G. E., Influence of the Exchange Screening Parameter on the Performance of Screened Hybrid Functionals. J. Chem. Phys. 2006, $125,224106$.

5. Henkelman, G.; Uberuaga, B. P.; Jonsson, H., A Climbing Image Nudged Elastic Band Method for Finding Saddle Points and Minimum Energy Paths. J. Chem. Phys. 2000, 113, 9901-9904.

6. Vineyard, G. H., Frequency Factors and Isotope Effects in Solid State Rate Processes. J. Phys. Chem. Solids 1957, 3, 121-127.

7. Henkelman, G. Methods for Calculating Rates of Transitions with Application to Catalysis and Crystal Growth. Ph.D. thesis, University of Washington, 2001, Chapter 4, Pages 60-69.

8. Hammer, B.; Hansen, L. B.; Norskov, J. K., Improved Adsorption Energetics Within Density-Functional Theory Using Revised Perdew-Burke-Ernzerhof Functionals. Phys. Rev. B 1999, 59, 7413-7421.

9. Grimme, S.; Antony, J.; Ehrlich, S.; Krieg, H., A Consistent and Accurate Ab Initio Parametrization of Density Functional Dispersion Correction (DFT-D) for the 94 Elements HPu. J. Chem. Phys. 2010, 132, 154104.

10. Wellendorff, J.; Lundgaard, K. T.; Mogelhoj, A.; Petzold, V.; Landis, D. D.; Norskov, J. K.; Bligaard, T.; Jacobsen, K. W., Density Functionals for Surface Science: ExchangeCorrelation Model Development with Bayesian Error Estimation. Phys. Rev. B 2012, 85, 235149.

11. Ramos, M.; Diaz, C.; Martinez, A. E.; Busnengo, H. F.; Martin, F., Dissociative and NonDissociative Adsorption of O-2 on $\mathrm{Cu}(111)$ and $\mathrm{Cu}-\mathrm{ML} / \mathrm{Ru}(0001)$ Surfaces: Adiabaticity Takes Over. Phys. Chem. Chem. Phys. 2017, 19, 10217-10221.

12. Sharada, S. M.; Bligaard, T.; Luntz, A. C.; Kroes, G. J.; Norskov, J. K., SBH10: A Benchmark Database of Barrier Heights on Transition Metal Surfaces. J. Phys. Chem. C 2017, 121, 19807-19815.

13. Marini, A.; Hogan, C.; Grunig, M.; Varsano, D., Yambo: an Ab Initio Tool for Excited State Calculations. Comp. Phys. Comm. 2009, 180, 1392.

14. Huang, J. K.; Pu, J.; Hsu, C. L.; Chiu, M. H.; Juang, Z. Y.; Chang, Y. H.; Chang, W. H.; Iwasa, Y.; Takenobu, T.; Li, L. J., Large-Area Synthesis of Highly Crystalline WSe2 Mono Layers and Device Applications. ACS Nano 2014, 8, 923-930.

15. Zheng, Y. J.; Huang, Y. L.; Chenp, Y. F.; Zhao, W. J.; Eda, G.; Spataru, C. D.; Zhang, W. J.; Chang, Y. H.; Li, L. J.; Chi, D. Z.; Quek, S. Y.; Wee, A. T. S., Heterointerface Screening Effects Between Organic Monolayers and Monolayer Transition Metal Dichalcogenides. ACS Nano 2016, 10, 2476-2484. 